\title{
Squeezing lubrication films: Layering transition for curved solid surfaces with long-range elasticity
}

\author{
B. N. J. Persson \\ IFF, FZ-Jülich, D-52425 Jülich, Germany \\ P. Ballone \\ IFF, FZ-Jülich, D-52425 Jülich, Germany and Dipartimento di Fisica, Instituto Nazionale per la Fisica \\ della Materia, Universitá degli Studi di Messina, Contrada Papardo, C.P. 50, 98166 Messina, \\ Italy
}

(Received 25 October 1999; accepted 9 March 2000)

\begin{abstract}
The properties of an atomic lubricant confined between two approaching solids are investigated by a model that accounts for the curvature and elastic properties of the solid surfaces. Well defined atomic layers develop in the lubricant film when the width of the film is of the order of a few atomic diameters. An external squeezing-pressure induces discontinuous, thermally activated changes in the number $n$ of lubricant layers. The precise mechanism for these layering transitions depends on $n$, and on the lubricant-surface pinning barriers. Thus, in the absence of sliding, unpinned or weakly pinned incommensurate lubricant layers give rise to fast and complete layering transitions. Strongly pinned incommensurate and commensurate layers give rise to sluggish and incomplete transformations, resulting in trapped islands. In particular, for commensurate layers it is often not possible to squeeze out the last few lubricant layers. However, lateral sliding of the two solid surfaces breaks down the pinned structures, greatly enhancing the rate of the layering transitions. In the case of sliding, an important parameter is the barrier for sliding one lubricant layer with respect to the others. When this barrier is larger than the lubricant-surface pinning barrier, the lubricant film tends to move like a rigid body with respect to the solid surface. In the opposite case, slip events may occur both within the lubricant film and at the lubricant-solid interface, making the squeeze-out process much more complex. In some of the simulations we observe an intermediate phase, forming immediately before the layering transition. This transient structure has a lower 2D density than the initial phase, and allows the system to release elastic energy, which is the driving force for the phase transformation. () 2000 American Institute of Physics.
\end{abstract}

[S0021-9606(00)70421-1]

\section{INTRODUCTION}

Sliding friction is one of the oldest problems in physics, and has undoubtedly a huge practical importance. ${ }^{1}$ In recent years, the ability to produce durable low-friction surfaces and lubricant fluids has become an important factor in the miniaturization of moving components in technologically advanced devices. For those applications, the interest is focused on the stability under pressure of thin lubricant films, since the complete squeeze out of the lubricant from an interface may give rise to cold-welded junctions, resulting in high friction and catastrophically large wear.

Recently, a large number of computer simulations and analytical studies of simple models have been presented, with the aim to gain insight into the atomistic origin of sliding friction. All the computer simulations we are aware of have used flat surfaces, represented by thin $(5-20 \AA)$ solid layers, which could not account for long range elastic effects (see, e.g., Ref. 2). However, all experiments related to boundary lubrication and sliding friction measured the properties of curved surfaces of mesoscopic or macroscopic dimensions, for which the elastic response to external forces is an essential feature. For example, in the surface forces apparatus, ${ }^{3}$ very thin mica sheets are glued onto two cylin- drical glass rods. By bringing the cylinders (rotated by $90^{\circ}$ relative to each other) in contact, a common interface is formed, whose shape and size is determined by the elastic deformation of the two solids. Curved surfaces are, of course, also involved in almost every real life sliding system, since even nominally flat surfaces have defects and asperities, and the contact between two macroscopic bodies will always occur in a number of discrete areas (typically of micrometer size), as illustrated in Fig. 1. For very smooth surfaces, the asperities will mainly deform elastically, i.e., negligible plastic deformation will occur.

In this work we introduce a model that takes into account the effect of long range elasticity, and we apply it to study the boundary lubrication for curved solid surfaces. In particular, we investigate the squeezing of molecular thin lubrication films, focusing on the nature of the $n \rightarrow n-1$ layering transition (where $n$ is the number of layers of lubrication atoms between the solid surfaces), that occurs with increasing applied pressure. In addition, we study the combined effect of squeezing and sliding. We find that, during sliding, the layering transition takes place at lower pressure than in the absence of sliding, i.e., it is easier to squeeze out the lubricant from the interfacial region when the two 


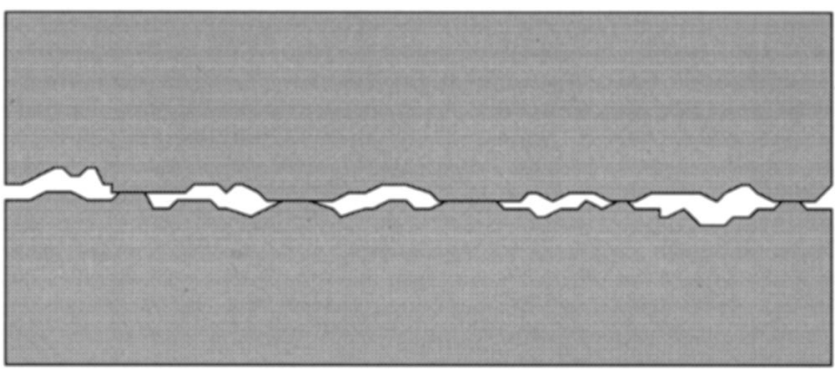

FIG. 1. Contact between two blocks with rough surfaces.

surfaces slide relative to each other. In another article, we shall apply the same computational method used in the present work to study sliding friction and boundary lubrication. $^{4}$

\section{QUALITATIVE DISCUSSION}

In order to highlight the global picture emerging from the simulation results reported below, we present in this section a qualitative discussion of the behavior of a lubricant layer wetting two approaching solid surfaces, and a summary of our simulation results.

We first consider two flat surfaces of finite extension, separated by a thick ( $\gg 100 \AA$ ) layer of a fluid lubricant (see Fig. 2). Under these conditions, an arbitrary small applied pressure is able to reduce the separation of the two solid surfaces, provided the lubricant can flow away from the region of closest approach. This process can be described by the Navier-Stokes equations, and one finds that the motion of a circular disk of diameter $D$, pushed towards a flat substrate by an applied pressure $P$, is described by the relation (see, e.g., Ref. 1)

$$
\frac{1}{h^{2}(t)}-\frac{1}{h^{2}(0)}=\frac{16 t P}{3 \mu D^{2}},
$$

where $h(t)$ is the time dependent separation between the two parallel surfaces, and $\mu$ is the viscosity of the fluid.

However, even for ideally flat surfaces, this formula typically breaks down when the separation of the two surfaces approaches the length scale set by $\sim 10$ times the diameter of the lubricant atoms or molecules. For smaller separations, the properties of the lubricant layer cannot be described by the macroscopic parameters characterizing the bulk fluid, since the solid surfaces induce layering in the

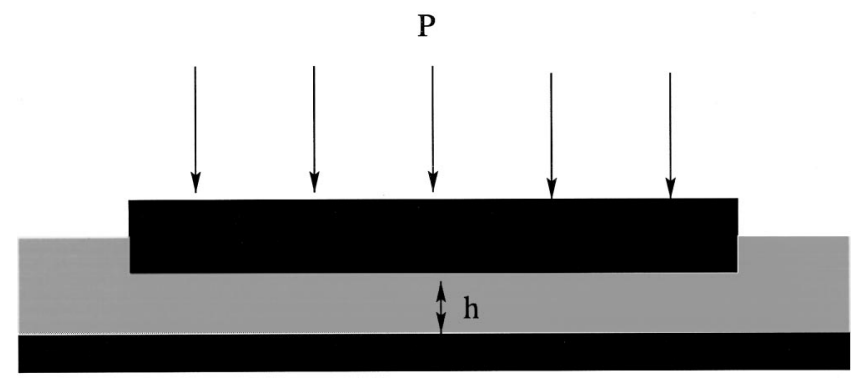

FIG. 2. A circular disk (diameter $D$ ) squeezed against a flat substrate in a fluid. direction away from the surfaces, and the corrugation of the solid walls induces a 2D-ordering in the nearest layers of lubricant molecules, thus modifying their response to the applied pressure. ${ }^{3,5,6}$ This ordering can be so strong that the lubricant film acquires a solid-like character, and develops a shear resistance that is at the origin of the static friction force observed even for lubricated interfaces. Finally, in the limit of very small separation between the two solid surfaces, specific lubricant-surface interactions dominate. For most lubricants, the binding to the solid surfaces is stronger than the intermolecular forces in the lubricant itself, and, as a consequence, it is often observed that it is difficult, or even impossible, to squeeze out the last one or two lubricant monolayers simply by increasing the perpendicular pressure. The difference in the binding free energy (per unit area), between a lubricant molecule bound to the solid surfaces and in the bulk liquid lubricant is quantified by the spreading pressure $p_{0}$ (see, e.g., Ref. 1).

It is important to notice that this qualitative picture is valid for almost every solid interface, even if no lubricant has been intentionally added, since most real surfaces are covered by organic contaminants which have an effect very similar to that of added lubricants.

In the case of two curved elastic surfaces, the lubricant in the region of closest approach is in contact with the low pressure reservoirs represented by the regions with larger separation. For molecular thin lubrication films, with increasing pressure the width of the lubricant film in the regions of closest approach is reduced by discontinuous steps, corresponding to the reduction in the number of $2 \mathrm{D}$ lubricant layers at the interface. The elimination of each layer starts with the nucleation of a $2 \mathrm{D}$ void that progressively grows by ejecting atoms into the low pressure regions. ${ }^{7}$ The void formation is a thermally activated process that can be described by concepts borrowed from classical nucleation theory. Consider, e.g., a double layer and assume that the lubricant is in a 2D-fluid state. Due to a thermal fluctuation, a small hole of radius $R$ can be formed in the lubrication film, as indicated in Fig. 3. The free energy cost to form the hole is $2 \pi R \Gamma$ $+\pi R^{2} p_{0}$, where the first term is due to the broken bonds at the periphery of the hole ( $\Gamma$ is a line tension) and the second term represents the work done against the spreading pressure $p_{0}$ in the film. On the other hand, the formation of the hole triggers the surface relaxation of the elastic solid, as illustrated in Fig. 3. It can be proved that the gain in elastic energy is proportional to $R^{3}$, thus leading to the following expression for the free energy change $U(R)$ associated to the void:

$$
U(R)=2 \pi R \Gamma+\pi R^{2} p_{0}-\alpha R^{3},
$$

where $\alpha \approx C P^{2} / E, E$ is the elastic modulus of the confining solids, and $C$ is a number of order unity (see Ref. 7). This function has the form shown in Fig. 4. Note that there is a barrier towards nucleating the layering transition, corresponding to the free energy cost $U\left(R_{c}\right)$ to form the critical nucleus of radius $R_{c}$, identified by the condition: $U^{\prime}\left(R_{c}\right)$ $=0$. The dependence of $\alpha$ on $P$ reported above implies that the barrier decreases with increasing pressure. For temperatures $T>0 \mathrm{~K}$, the nucleation of the layering transition occurs 
$\mathbf{P}$

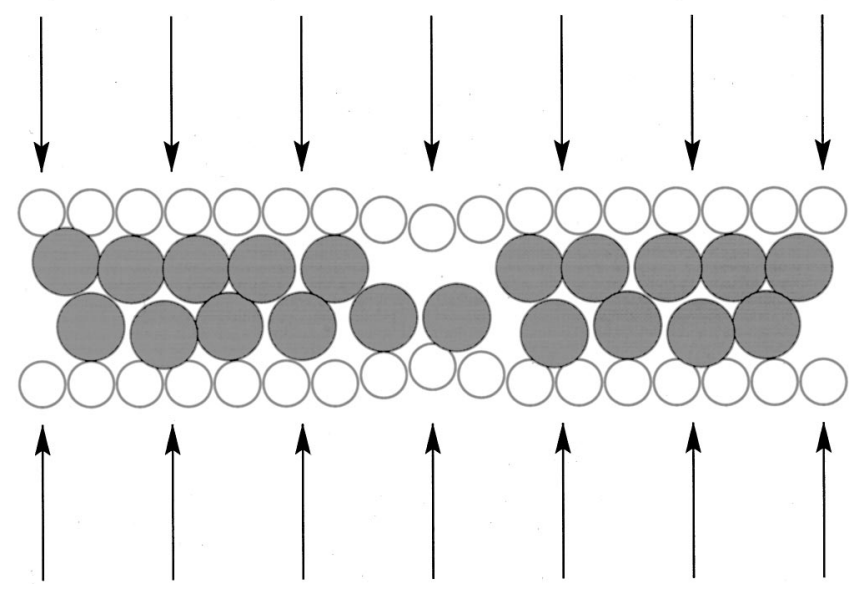

FIG. 3. Interface between two elastic solids separated by a lubricant bilayer. The solids are squeezed together by the pressure $P$. The gray particles denote the lubricant atoms and the unfilled circles the bottom and top layers of atoms of the block and the substrate, respectively. Note the relaxation of the elastic solids into the small void: this state represents the critical nucleus for the layering transition $n=2 \rightarrow 1$.

via a thermal fluctuation, with a rate $w$ given by $w$ $=w_{0} \exp \left[-U\left(R_{c}\right) / k_{B} T\right]$, where the prefactor $w_{0}$ is determined by the nature of the thermal fluctuations leading to the void (see Ref. 7).

We note that in many practical situations the nucleation of the layering transition may occur at some "weak" point between the surfaces where imperfections, e.g., foreign adsorbates (like water or some organic contamination), may locally reduce the spreading pressure (which can even become negative, i.e., nonwetting). This has been observed in some experiments where the layering transitions start repeatedly at the same point in the contact area. ${ }^{8}$ This situation is obviously similar to that for three-dimensional systems, where the formation of a new phase, e.g., solidification of an undercooled liquid, usually starts at "impurities" or at other anomalous points (dust particles, ions, surfaces, etc.).

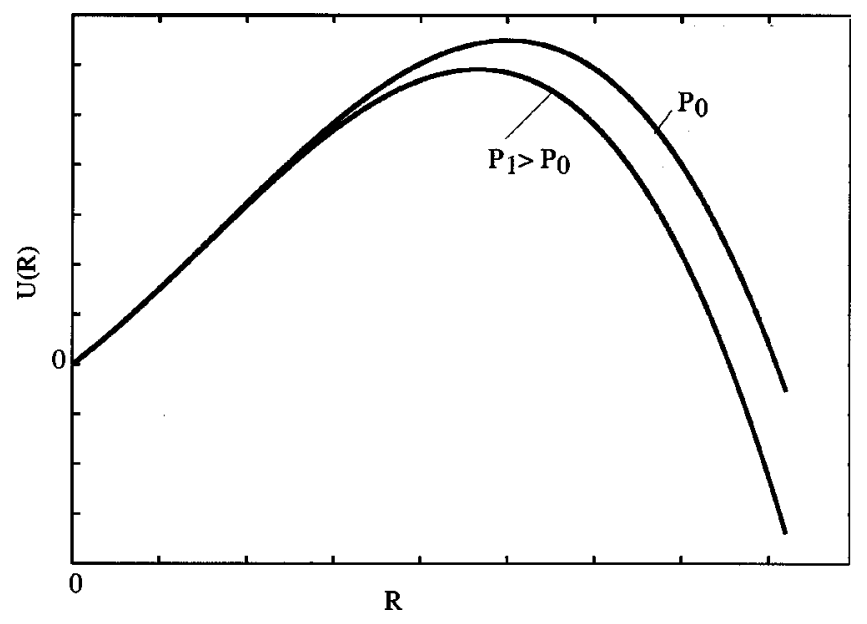

FIG. 4. The dependence of the free energy $U(R)$ on the radius $R$ of the void. Note that the barrier height for nucleating the layering transition decreases with increasing pressure. (a)

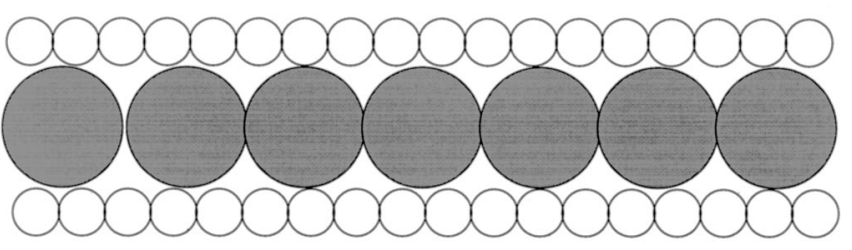

Incommensurate, unpinned or weakly pinned

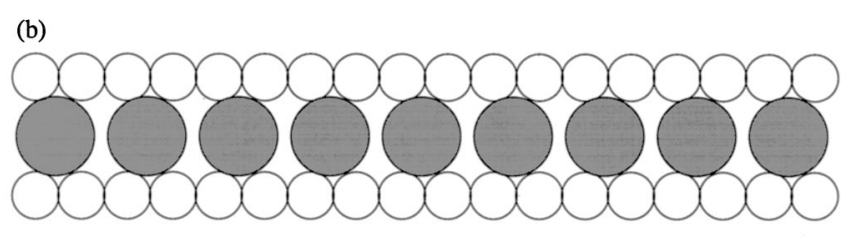

Commensurate, strongly pinned

FIG. 5. Incommensurate and commensurate lubricant monolayers at the interface between two solids.

The assumption of a $2 \mathrm{D}$ fluid state for the lubricant layer is often not justified in practice, as indicated by a nonvanishing static friction force measured at most interfaces. Instead, as discussed above, the lubrication film is partly or entirely in a solid-like state, which can be either commensurate or incommensurate with the underlying solid surfaces (see Fig. 5), and sometimes it is in a glassy state. Even in these cases, the thinning of the interface occurs in steps, and the layering transition starts by the nucleation of a small "hole" (stressaided activated process). However, the squeeze-out kinetics depends on the precise state of the lubricant layers. For solid surfaces separated by unpinned or weakly pinned (incommensurate) lubrication layers, fast and complete layering transitions occur. Commensurate or strongly pinned incommensurate layers lead to sluggish and incomplete transitions, possibly leaving islands trapped in the contact region. In fact, for commensurate layers we observe that it is nearly impossible to squeeze out the last few layers simply by increasing the perpendicular pressure. However, the squeeze-out rate is enhanced by lateral sliding, since, in this case, the lubricant film can turn into a disordered or fluid state, facilitating the ejection of an entire layer.

In our study, we simulate the late stages of the approach of two solid surfaces, wetted by an atomic lubricant (whose parameters are modeled on $\mathrm{Xe}$ ), and forming a curved interface. Our simulation reproduces the step-like evolution of the parameters characterizing the interface, corresponding to the discontinuous change in the number $n$ of lubricant layers in the region of shortest separation.

We observe that the nucleation of the voids, leading to the $n \rightarrow n-1$ transition, occurs by a thermally activated process, and we study the dependence of the transformation kinetics on the corrugation of the two solid surfaces, on temperature, and on the relative perpendicular and transversal (sliding) velocity of the two solid surfaces. In particular, we simulate three different types of solids, characterized by different surface corrugation, giving rise to two incommensurate hexagonal lubricant layers [case (A) and (B)], and to a commensurate interface $[$ case $(\mathrm{C})]$. 


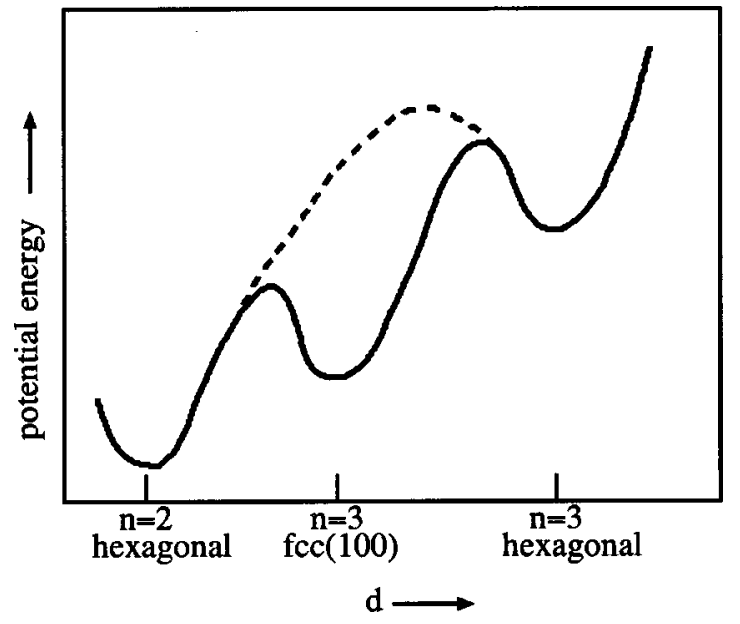

FIG. 6. The free energy as a function of the separation $d$ between the solid surfaces at the interface (schematic).

In some cases, immediately before the layering transition, we observe the formation of a transient phase in the lubrication film, which has the effect of reducing the number of atoms in the region of closest contact, and opens the way for the $n \rightarrow n-1$ layering transition. Thus, in model A and $\mathrm{B}$ studied below the Xe atoms normally form incommensurate hexagonal layers for which the concentration of atoms within the planes is maximal, with, however, a relatively large separation between the planes. In this case we observe a transition from hexagonal to fcc(100) planes, conserving the number of lubricant layers. Since the density of Xe atoms in the fcc(100) layers is smaller than in the original hexagonal planes, this phase transformation reduces the number of lubricant atoms in the high pressure contact region, and enhances the mobility of the atoms. Moreover, the separation between the fcc(100) planes is smaller than between the original hexagonal layers, and the solid surfaces move closer to each other. As a result, the observed transformation reduces the elastic energy of the system. On the other hand, the decrease of the number of lubricant molecules in contact with the solid walls [as a result of the lower 2D concentration of Xe atoms in the fcc(100) layers] implies a loss of $\mathrm{Xe}$-solid adsorption energy. The balance between these two opposite tendencies depends on the strength of the applied pressure: for sufficiently high pressure the transformation has to take place, provided it is not preempted by the layering transition. We observed that the nucleation of the $n \rightarrow n-1$ transition closely follows the appearance of the fcc(100) structure, probably because the decrease of the 2D Xe density favors the fluctuations leading to the $(n-1)$ "critical hole." Once it is formed, this hole grows rapidly, while the remaining $(n-1)$ fcc $(100)$ layers revert back to the hexagonal structure.

Figure 6 illustrates schematically the variation of the free energy as a function of the separation $d$ between the solid surfaces. The solid and dashed lines correspond to the case when the film undergoes the transformation discussed above, and when it is inhibited, respectively.

A second interesting result of our simulation is the observation (recently reproduced in experiments ${ }^{9}$ ) of an incom-
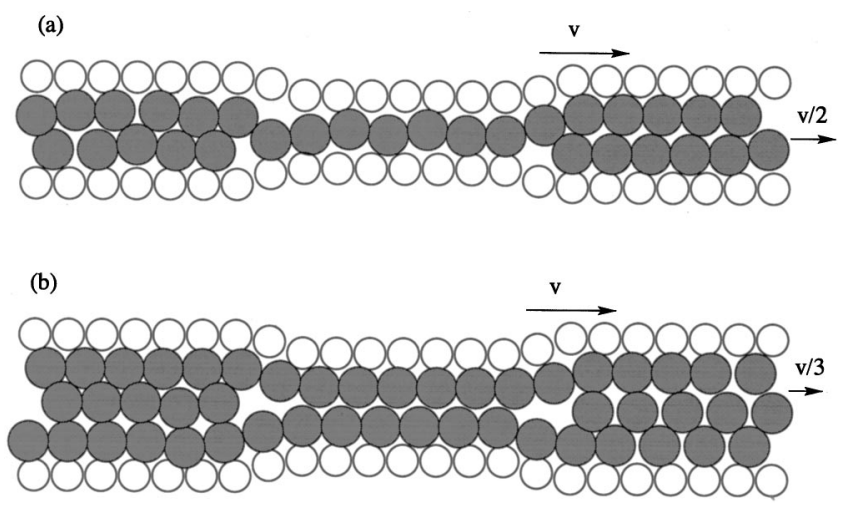

FIG. 7. If the lubricant film experiences a weak pinning force at the solidlubricant interface, then during squeeze-out the lubricant film moves as a rigid unit with a velocity which, for the bilayer and trilayers, is half and one-third of the squeeze-out front velocity, respectively.

plete squeeze-out resulting in 2D islands trapped between the approaching surfaces. Thus, as an example, we describe below a pinned incommensurate Xe film displaying an incomplete $n=2 \rightarrow 1$ transition, leaving behind a trapped $n=2$ island surrounded by the $n=1$ area. We point out that we never observed such an incomplete transition in the case in which lateral sliding is superimposed to squeezing: in this case the effect of pinning is reduced. In general, in our simulations we observe that it is much easier to squeeze out the lubrication films during sliding and squeezing, as compared to only squeezing. This observation is also in agreement with experiment.

Finally, let us comment on the influence on intralubricant forces, and, in particular, on the importance of the free energy barriers for sliding the lubricant layers with respect to each other. First, we observe that these barriers tend to be large, because all the lubricant atoms have the same size, and therefore, the lubricant layers are commensurate and pinned to each other. When these lubricant-lubricant sliding barriers are larger than the barriers to slide the lubricant on the solid surfaces, we find that during the combined squeeze-out and sliding there is no slip between the lubricant layers, but the total slip occurs only at the solid-lubricant interfaces. In other words, the lubricant film tends to move as a single unit relative to the solid surfaces. For the model we study below, this implies that during the $n=2 \rightarrow 1$ squeeze-out, if the $n$ $=1$ area expands in the positive (negative) $x$-direction with the velocity $v$, then the bilayer moves to the right with the speed $v=1 / 2$ and there is no slip between the two layers (see Fig. 7). Similarly, if we consider the $n=3 \rightarrow 2$ transition, the trilayers have to move with the speed $v / 3$. This behavior is observed for models (A) and (B) below (where the adsorbates form incommensurate layers which are unpinned or weakly pinned by the substrate), but not for model (C) (where a commensurate layer is formed) where the squeezeout (which now only occur during sliding) is much more complex.

\section{MODEL}

We are concerned with the properties of a lubricant film squeezed between the curved surfaces of two elastic solids. 


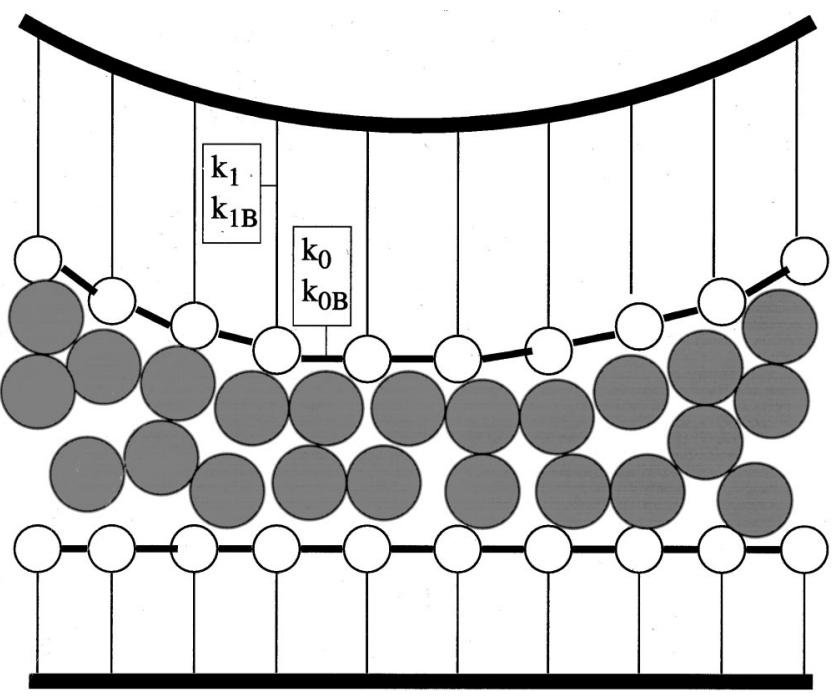

FIG. 8. Schematic picture of the central region of the squeezing model used in the present article.

In experiments, a system of this type is obtained by gluing two elastic slabs (of thickness $W_{1}$ and $W_{2}$ ) to "rigid" surface profiles of arbitrary shape. If the radius of curvatures of the rigid surfaces are large compared to $W_{1}$ and $W_{2}$, the elastic slabs will deform, reproducing with their free surfaces the (nearly arbitrary) shape of the underlying rigid profiles.

To account for the elastic response of the slabs, without dealing with the large number of atoms required to simulate a mesoscopic elastic solid, in our model we treat at the atomistic level only the last few atomic layers of the solids at the interface. The force constants connecting these atoms to the underlying solid, however, are not the bare parameters, determined by the model interatomic potential. Instead, those force constants are treated as effective parameters that implicitly reintroduce the elastic response of the slabs of arbitrary width $W_{1}$ and $W_{2}$.

The resulting model is illustrated in Fig. 8. The atoms in the bottom layer of the block (open circles) form a simple square lattice with lattice constant $a$, and lateral dimension $L_{x}=N_{x} a$ and $L_{y}=N_{y} a$. In the following, periodic boundary conditions are assumed in the $x y$ plane. The atoms interact with each other via "stiff" springs (thick lines) of bending force constant $k_{0 B}$ and stretching force constant $k_{0}$. Moreover, each atom is connected to the upper rigid surface profile by "soft" elastic springs, of bending force constant $k_{1 B}$ and stretching force constant $k_{1}$.

The numerical value of all these force constants $k_{0}, k_{0 B}$, $k_{1}$, and $k_{1 B}$ are determined in such a way to mimic the elastic response of the entire slab. If we apply a shear stress $\sigma$ to the slab, the resulting strain $\epsilon$ is given by $\sigma=2 G \epsilon$, where $G=E / 2(1+\nu)$ is the shear modulus, $E$ is the elastic modulus, and $\nu$ the Poisson ratio. If we write the shear strain as $\epsilon=\Delta / 2 a$ then $\sigma=k_{0 B} \Delta / a^{2}=G \Delta / a$ and we get $k_{0 B}$ $=G a$. Similarly, we obtain that $k_{0}=E a$. Next, let us consider an elastic slab of thickness $W$. If we apply a shear stress $\sigma$, we get the relative displacement $x$ so that the strain is $\epsilon=x / 2 W$. Thus $\sigma=G x / W$ which must equal $k_{1 B} x / a^{2}$ and hence $k_{1 B}=G a^{2} / W$. In a similar way one can obtain $k_{1}$
$=E a^{2} / W$.

The substrate is treated in a similar way as the block. In our simulations, we shall assume that the block is moving, while the bottom surface of the substrate if fixed in space.

Between the block and substrate we assume a layer (monolayer or more) of lubrication atoms, which interact with each other via Lennard-Jones pair potentials:

$$
v(r)=4 \epsilon_{0}\left[\left(\frac{r_{0}}{r}\right)^{12}-\left(\frac{r_{0}}{r}\right)^{6}\right] .
$$

The parameters $\left(\epsilon_{0}, r_{0}\right)$ have been chosen to describe Xe. We also assume that the lubrication atoms interact with the atoms of the solid surfaces via Lennard-Jones pair potentials but with different parameters $\left(\epsilon_{1}, r_{1}\right)$. In the Appendix we present the basic equations we have used in the computer simulations.

In the simulations presented below we assume that the elastic properties of the solids correspond (approximately) to steel. That is, we use $E=1 \times 10^{11} \mathrm{~N} / \mathrm{m}^{2}$ (elastic modulus), $\nu$ $=0.3$ (Poisson ratio), and $\rho=5096 \mathrm{~kg} / \mathrm{m}^{3}$ (mass density). The block is $100 \AA$ thick and has a cosine corrugation along the $x$ direction, while the substrate is flat and consists of just one monolayer of atoms. The parameters for the interaction among the lubricant atoms $\left(\epsilon_{0}=20 \mathrm{meV}, r_{0}=4 \AA\right.$, and the atomic mass 100) correspond to Xenon.

Computations have been done for three different cases:

(A) $r_{1}=1.087 r_{0}=2 a$,

(B) $r_{1}=1.1 r_{0}=1.375 a$,

(C) $r_{1}=1.1 r_{0}=a$,

where $a$ is the common lattice constant of the block and the substrate. In all three cases we assume $\epsilon_{1}=3 \epsilon_{0}=60 \mathrm{meV}$. In models (A) and (B), we adopt $N_{x}=200, N_{y}=30$, while for model (C) we use $N_{x}=150, N_{y}=25$.

Figure 9 shows the potential energy (in eV) (top) and the equilibrium height (in units of the substrate lattice constant a) (bottom) for a Xe atom displaced over the substrate from an on-top site, over the hollow site, to another on-top site (see inset). The curves denoted by (A), (B), and (C) are for the three different adsorbate-substrate interactions described above. The Xe atoms binds strongest in the hollow sites and weakest in the on-top sites. The binding energy in the hollow site is $E_{B}=0.91,0.46$, and $0.3 \mathrm{eV}$ for cases (A), (B), and $(\mathrm{C})$, respectively, and the overall corrugation in the binding potential energy surface equals $1.2 \%, 13 \%$, and $39 \%$, respectively. The fluctuation in the height of the Xe atom between the hollow and on-top site is $0.012 a, 0.08 a$, and $0.2 a$ in cases (A), (B), and (C), respectively. We note that when an adsorbate layer is confined at high pressure between two solid surfaces, the effective barrier for diffusion will strongly increase. Nevertheless, even in this case, the qualitative difference between the cases (A), (B), and (C) exhibited in Fig. 9 remain unchanged.

At zero temperature, the lubricant atoms form either incommensurate adsorbate structures [case (A) and (B)] [see Fig. 10(a); the block and substrate atoms are not shown] or a commensurate $(1 \times 1)$-structure [case (C)] [see Fig. 10(b); the adsorbates occupies the hollow sites on the substrate]. 

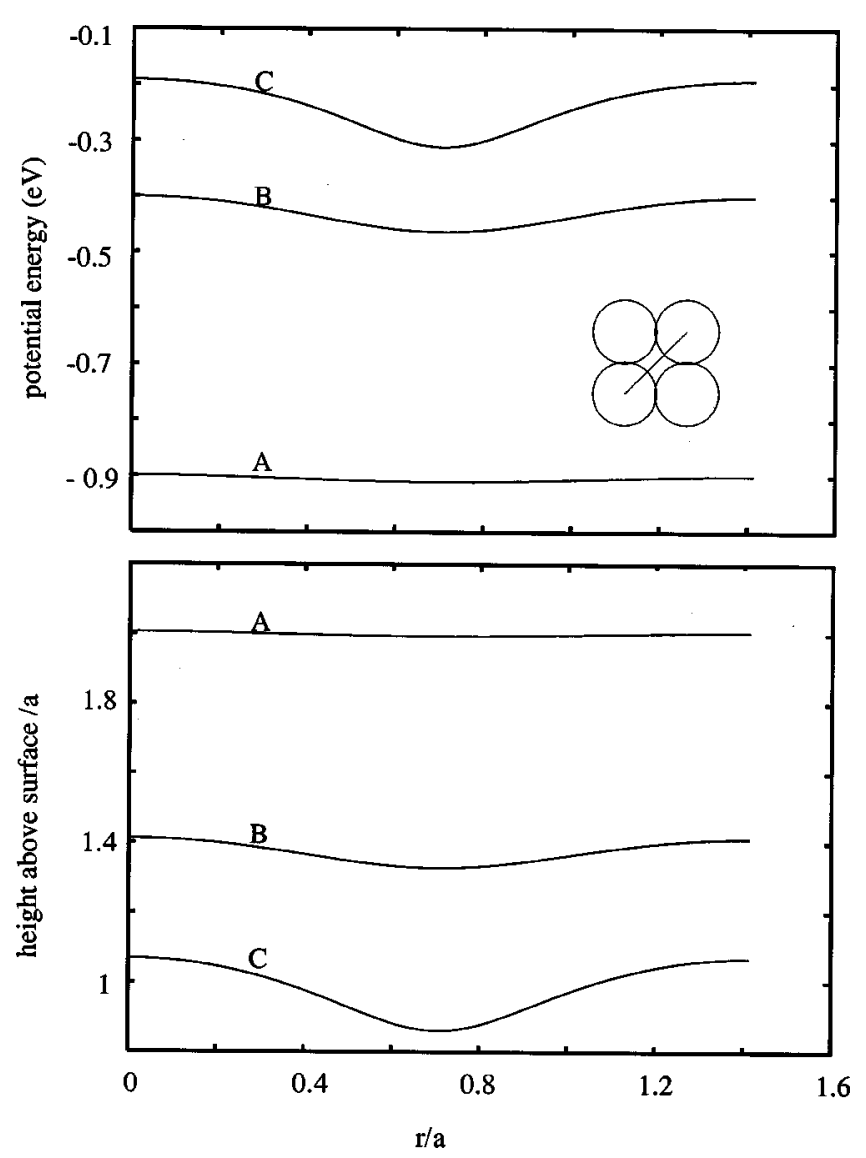

FIG. 9. The potential energy (top) and the height above the surface (bottom) as a function of the lateral position of the Xe atom between ontop-hollowontop (see inset). The curves (A), (B), and (C) correspond to three different models (see text).

An incommensurate adsorbate layer is either pinned or unpinned. It has been shown by Aubry ${ }^{10}$ that pinning occurs if the amplitude $\Delta E$ of the lateral corrugation of the adsorbate-substrate interaction potential is larger than an effective elastic energy $\mathcal{E}$ associated with the incommensurate solid layer. Thus, there are two distinct types of incommensurate configurations, a pinned state for $\mathcal{E} / \Delta E \ll 1$ and an unpinned state for $\mathcal{E} / \Delta E \gg 1$, and the transition between them (as a function of $\mathcal{E} / \Delta E$ ) is associated with the continuous phase transition known as "the breaking of analyticity." In (a)

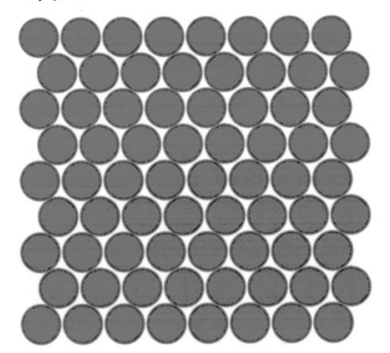

(b)

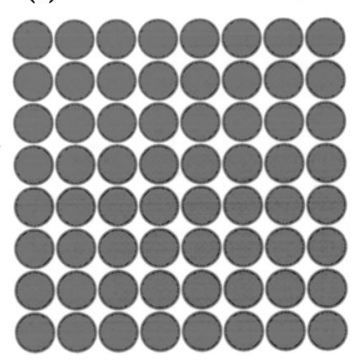

FIG. 10. High-coverage and low-temperature structures of the lubricant atoms on the substrate. Only the lubricant atoms are shown. (a) is an incommensurate structure formed in models (A) and (B) while (b) is the commensurate $(1 \times 1)$ structure formed in model $(\mathrm{C})$ (lubricant atoms occupy the hollow sites). See text for details.
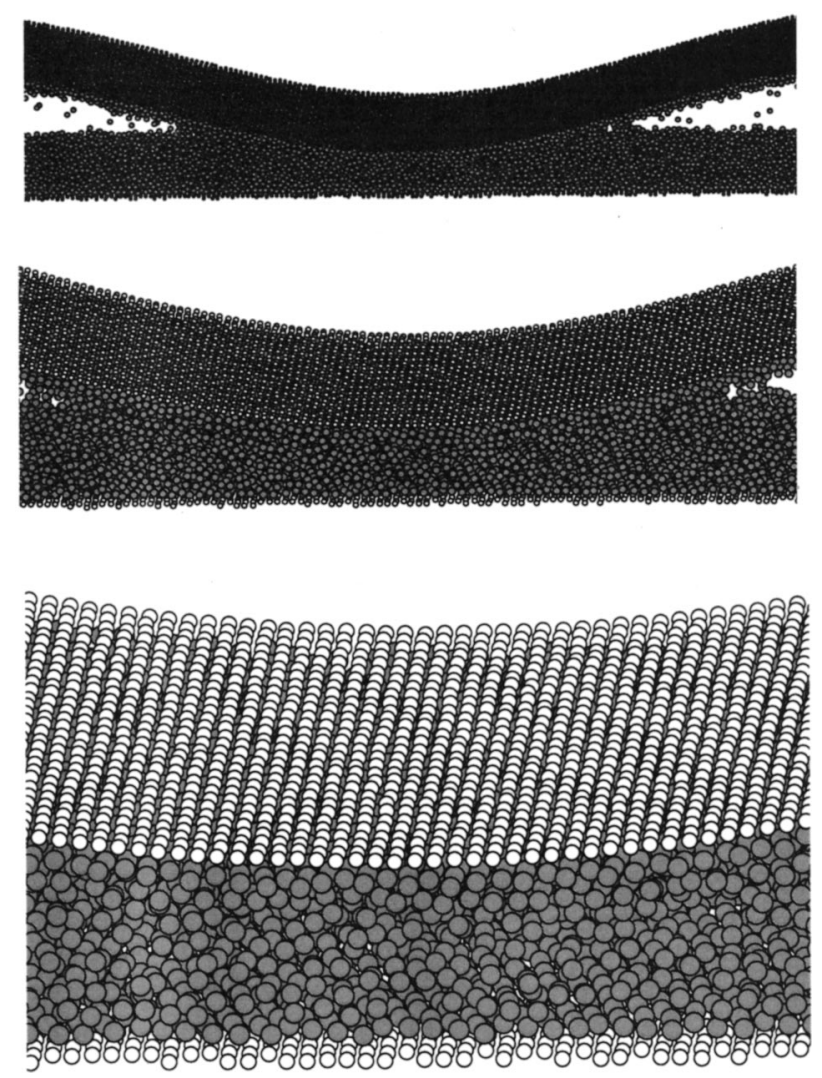

FIG. 11. Snapshot pictures of the central region of the junction. The system is shown with increasing magnification at an inclined view angle. In the bottom figure the gray particles are the lubrication atoms $(\mathrm{Xe})$ while the unfilled circles are the bottom layer of atoms on the block and the top layer of atoms of the substrate.

contrast to the periodic substrate potential, one can show that randomly distributed point defects always give rise to pinning, but we will assume a perfect surface in the following discussion. In the present case (in the absence of confining pressure), the incommensurate structures are unpinned in case (A) and pinned in case (B).

Figure 11 shows three pictures of increasing magnification of the model used in the computer simulations. The open circles in the bottom figure show the top layer of atoms of the substrate (flat surface) and the bottom layer of atoms on the block (curved surface). During most of the simulations the rigid upper surface of the block moves towards the rigid lower surface of the substrate with a constant velocity $v_{z}$.

Figure 12 reports the instantaneous perpendicular stress acting on the surface of the substrate at the end of the $T$ $=50 \mathrm{~K}$ simulation shown in Fig. 14 [case (A)]. Note that the stress is maximal at the center of the contact area. The (large) fluctuations in the stress result from the irregular thermal motion of the lubrication atoms, and would disappear if the stress is averaged over a sufficiently long time. The contact between the block and the substrate extends approximately between $-40 a<x<40 a$, where $a$ is the substrate lattice constant.

\section{SIMULATION RESULTS}

The general description of the interface behavior reported on in Sec. II is complemented in this section by a 


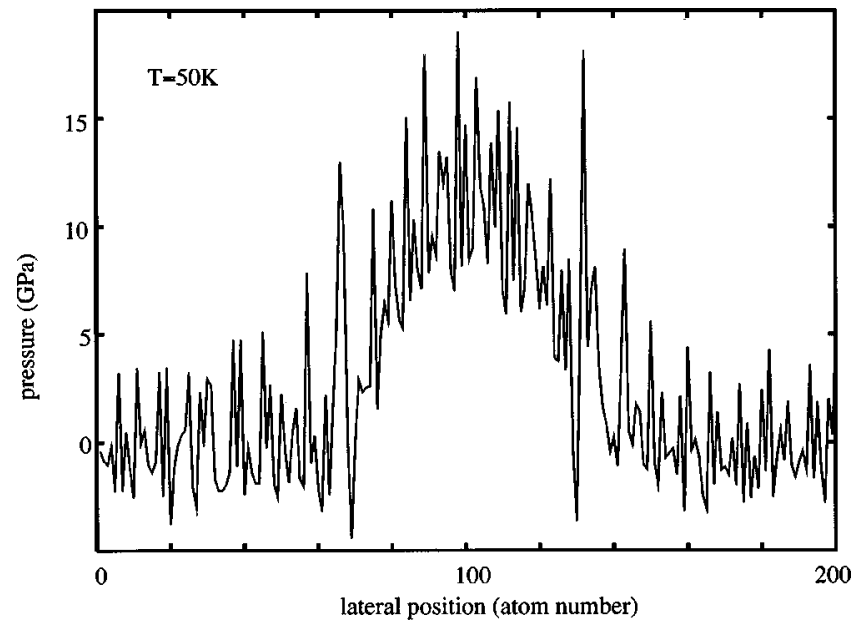

FIG. 12. The instantaneous pressure distribution acting on the substrate (or the block) at the block-substrate interface. The snapshot is obtained at the end of the $T=50 \mathrm{~K}$ simulation shown in Fig. 14.

series of figures, illustrating the major points discussed in Sec. II. We report separately the results for the three cases (A), (B), and (C) introduced in Sec. III.

\section{A. Incommensurate unpinned layer}

We consider first case (A), where the Xe monolayer forms an incommensurate structure. The parameters relevant for this case were given above and the (monolayer) equilibrium structure in the absence of an applied shear force is shown in Fig. 10(a). Note that for a single atom adsorbed on the substrate the binding energy is highest in the hollow sites $\left(E_{B}=0.91 \mathrm{eV}\right)$ and only $1.2 \%$ smaller in the on-top sites, so that the lateral corrugation of the ground state potential energy surface in the present case equals $\Delta E=11 \mathrm{meV}$. Since the substrate lattice constant $a=2.174 \AA$ is much smaller than the $\mathrm{Xe}-\mathrm{Xe}$ equilibrium separation (which is close to $r_{0}=4 \AA$ ), the incommensurate structure shown in Fig. 10(a) is indeed expected, and, in the absence of confining pressure, the adsorbate layer is unpinned.

Figure 13 shows the average perpendicular stress (or pressure) acting on the substrate (or block) as a function of the displacement of the block towards the substrate for $T$ $=300 \mathrm{~K}$. The block and the substrate are initially separated by about four Xe monolayers. Calculations are presented for two different squeeze velocities, $v_{1} \approx 6 \mathrm{~m} / \mathrm{s}$ and $v_{2} \approx 3 \mathrm{~m} / \mathrm{s}$, where (for clarity) the latter curve is displaced towards negative pressure by $0.2 \mathrm{GPa}$. The initial separation is close to contact and a small wetting bridge is formed between the two surfaces, leading to a weak attraction. The three "bumps" on the curves correspond to the layering transitions (with increasing pressure) $n=4 \longrightarrow 3,3 \rightarrow 2$, and $2 \rightarrow 1$. Note that these transitions are rather abrupt, and that the pressure drops as a result of the squeeze-out of each $\mathrm{Xe}$ monolayer. This drop is due to the fact that the layering transitions occur so rapidly that the upper surface moves only a small fraction of the diameter of the Xe monolayer during the squeeze-out of one layer; thus the elastic solid (block) can expand (relax) downwards so that the elastic stress in the block (and hence the pressure at the interface) is

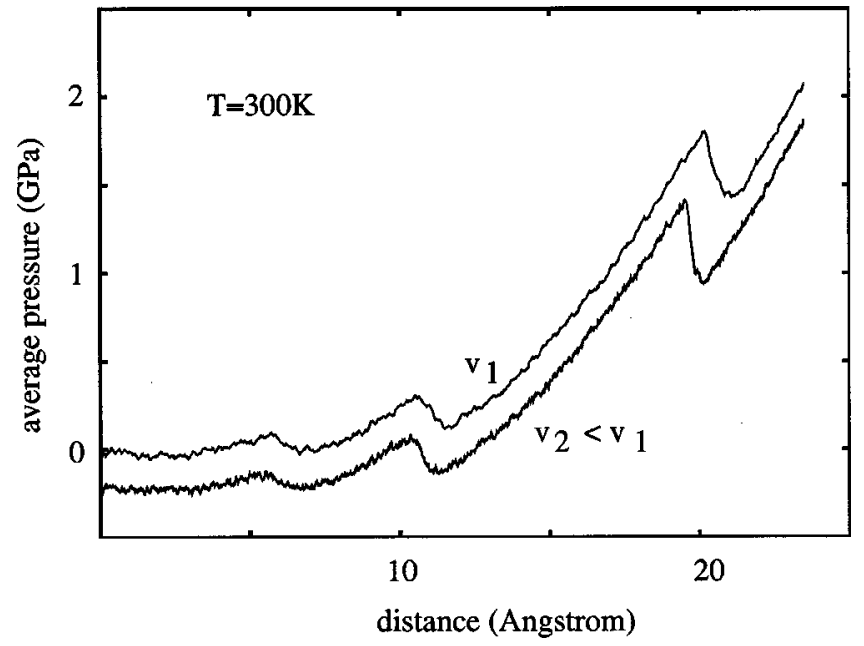

FIG. 13. The dependence of the average pressure on the distance the upper surface of the block has moved towards the bottom surface of the substrate. Results are shown for two different squeezing velocities $v_{1} \approx 6 \mathrm{~m} / \mathrm{s}$ and $v_{2}$ $\approx 3 \mathrm{~m} / \mathrm{s}$. For model (A) at $T=300 \mathrm{~K}$.

reduced. Note that the layering transitions occur at lower pressures for the lower-velocity $v_{2}$ case: this suggests that the squeeze-out is a thermally activated process.

Figure 14 (top) shows the same as in Fig. 13 for the
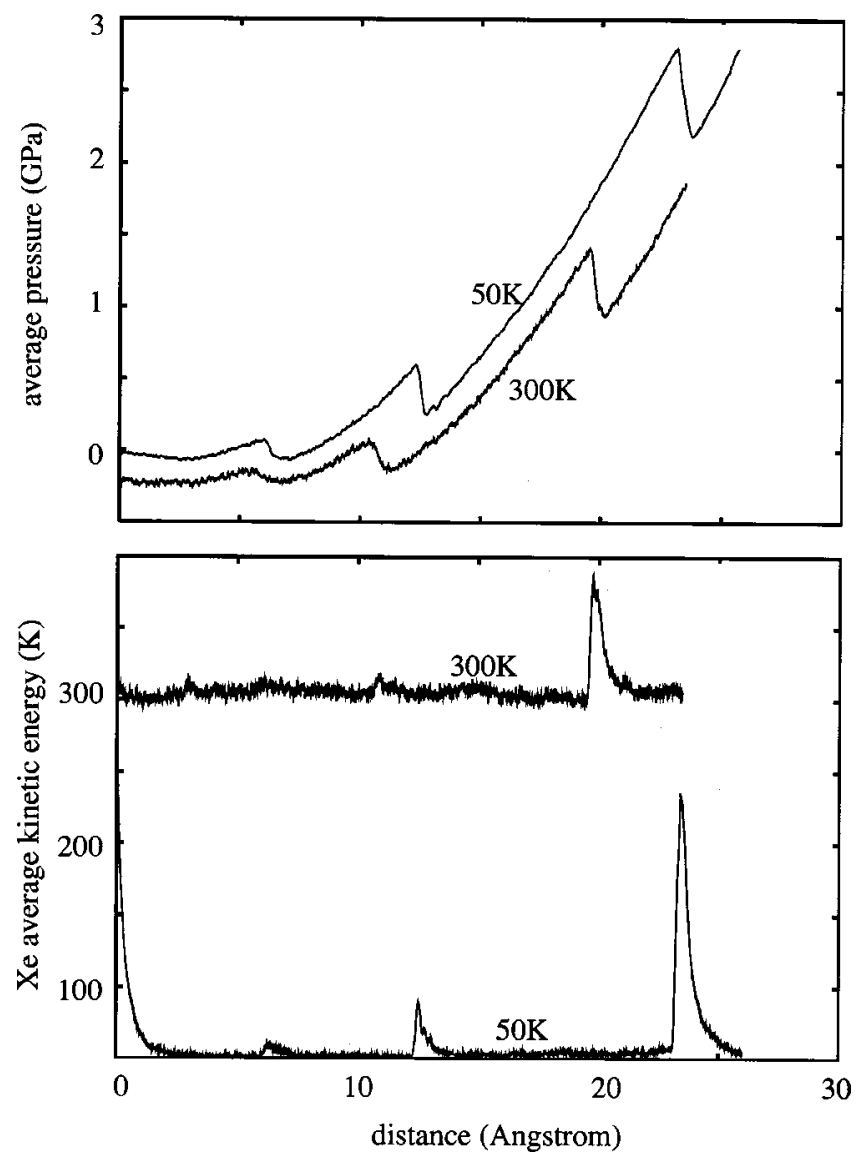

FIG. 14. The dependence of the average pressure (top) and the average Xe kinetic energy (bottom) on the distance the upper surface of the block has moved towards the bottom surface of the substrate. Results are shown for two different temperatures $T=50 \mathrm{~K}$ and $T=300 \mathrm{~K}$, and with the squeeze velocity $v_{2} \approx 3 \mathrm{~m} / \mathrm{s}$. For model (A). 


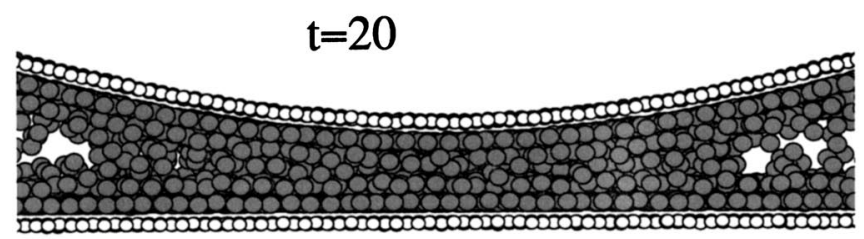

70

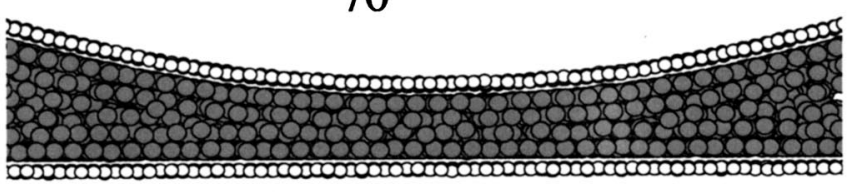

80

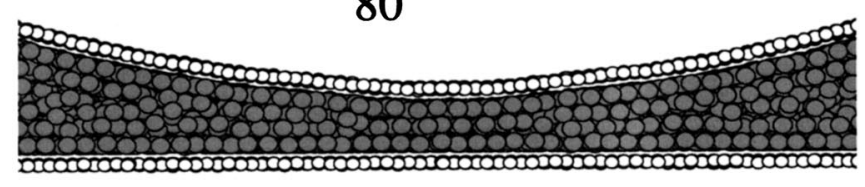

120

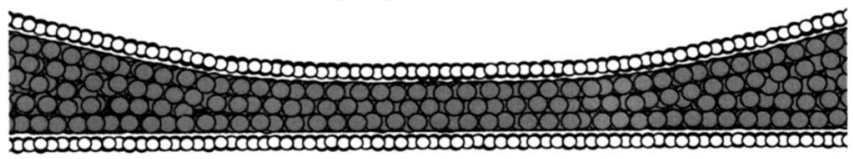

130

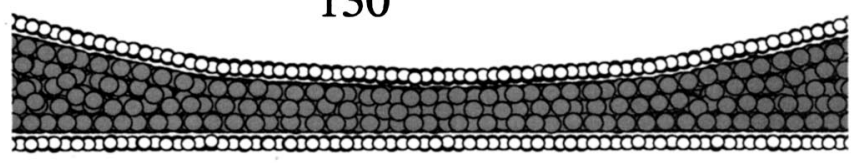

140

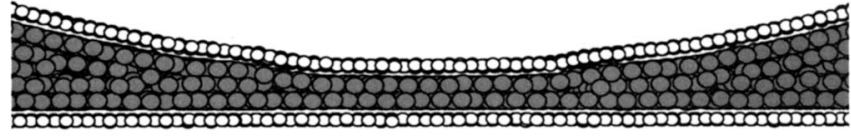

150

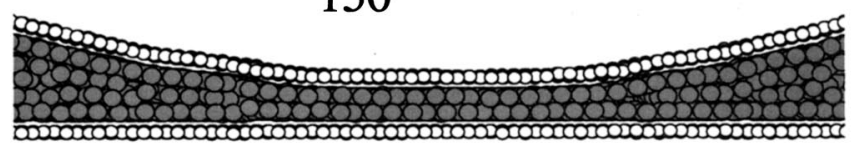

240

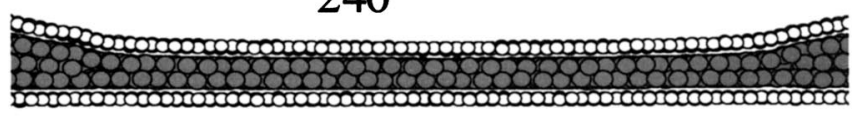

250

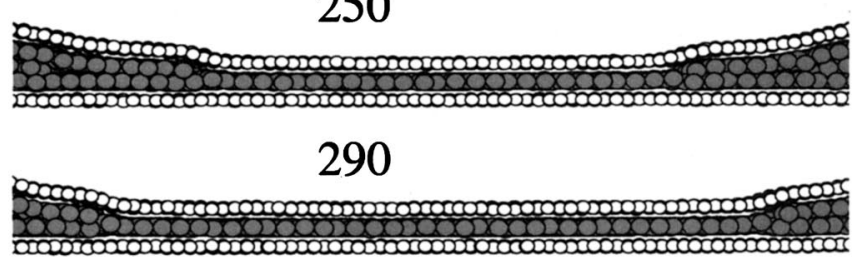

FIG. 15. Snapshot pictures during squeeze-out. The time of each snapshot is indicated. For model (A) at $T=300 \mathrm{~K}$.

squeeze velocity $v_{z} \approx 3 \mathrm{~m} / \mathrm{s}$, and for two different temperatures, $T=50$ and $300 \mathrm{~K}$. The bottom figure shows the $\mathrm{Xe}$ average kinetic energy in temperature units $(\mathrm{K})$. The $T$ $=50 \mathrm{~K}$ simulations actually started with the $T=300 \mathrm{~K}$ thermal equilibrium configuration, but this has no influence on the layering transitions as the temperature in the lubrication film (and the solid surfaces) has reached $T=50 \mathrm{~K}$ long time before the first $(n=4 \rightarrow 3)$ transition occurred (see figure). The layering transitions occur at higher pressures for lower temperatures indicating that it is a thermally activated process. Note that at the lowest temperature the lubrication film picks up more kinetic energy during the squeeze-out of the monolayers than at room temperature: this is, at least in part, a result of the higher pressure which acts at the start of squeeze-out in the former case.

Figure 15 shows a sequence of snapshot pictures of the central interface region during squeezing at $T=300 \mathrm{~K}$. The upper surface of the block moves towards the bottom surface of the substrate with the velocity $v_{2} \approx 3 \mathrm{~m} / \mathrm{s}$. Note that at high pressures the block deforms elastically, forming a flat area separated from the substrate by a few well-defined Xe layers. We emphasize that this effect is missing in any model that does not account for the long range elasticity of the interface.

Figure 16 shows pictures of the central part of the lubrication layer for the same system as in Fig. 15, as seen from the direction of the applied external pressure. Because of the high temperature $(T=300 \mathrm{~K})$ the lubrication film is initially condensed in a liquid state on the solid surfaces (snapshot $t$ $=20$ ): note the local hexagonal structure (as expected for a
2D Lennard-Jones fluid) but no long range order occurs. Immediately before the layering transition $n=3 \rightarrow 2$ (snapshot $t=130$ ), the lubrication film in the central region undergoes a phase transformation and now exhibits fcc(100) planes parallel to the solid surfaces. Since the fcc(100) plane has a lower concentration of $\mathrm{Xe}$ atoms than the hexagonal layer (assuming the same nearest-neighbor $\mathrm{Xe}-\mathrm{Xe}$ distance), a fraction of the Xe solid binding energy is lost during this transformation. On the other hand, the solid surfaces can now move closer to each other, since the distance between the fcc(100) layers is smaller than between the hexagonal layers, and in this way elastic energy is gained. After the phase transformation, the layering transition $n=3 \rightarrow 2$ can occur much more easily, since density fluctuations (opening up of a "hole") requires less energy in the more dilute fcc(100) layers than in the higher density hexagonal layers. Thus the transition $n=3 \rightarrow 2$ occurs rapidly after the transformation into the fcc(100) layer structure. After the $n=3$ $\rightarrow 2$ transition, a nearly perfect hexagonal $\mathrm{Xe}$ bilayer is formed (see snapshot $t=140$ ). It is also clear from the figure that at these high confining pressures the amplitude of the thermal motion of the lubrication atoms is strongly reduced, and the lubrication film appears to be in a solid state even at $T=300 \mathrm{~K}$, a temperature at which it is likely to be in a liquid state at lower pressure (compare with snapshot at $t=20$ ). After the transition $n=2 \rightarrow 1$ a single well-ordered hexagonal Xe monolayer is formed $(t=290)$.

Let us estimate the local pressure necessary for the [hexagonal $\rightarrow \mathrm{fcc}(100)]$ phase transformation. Let us denote the nearest-neighbor separation between the Xe atoms in the 

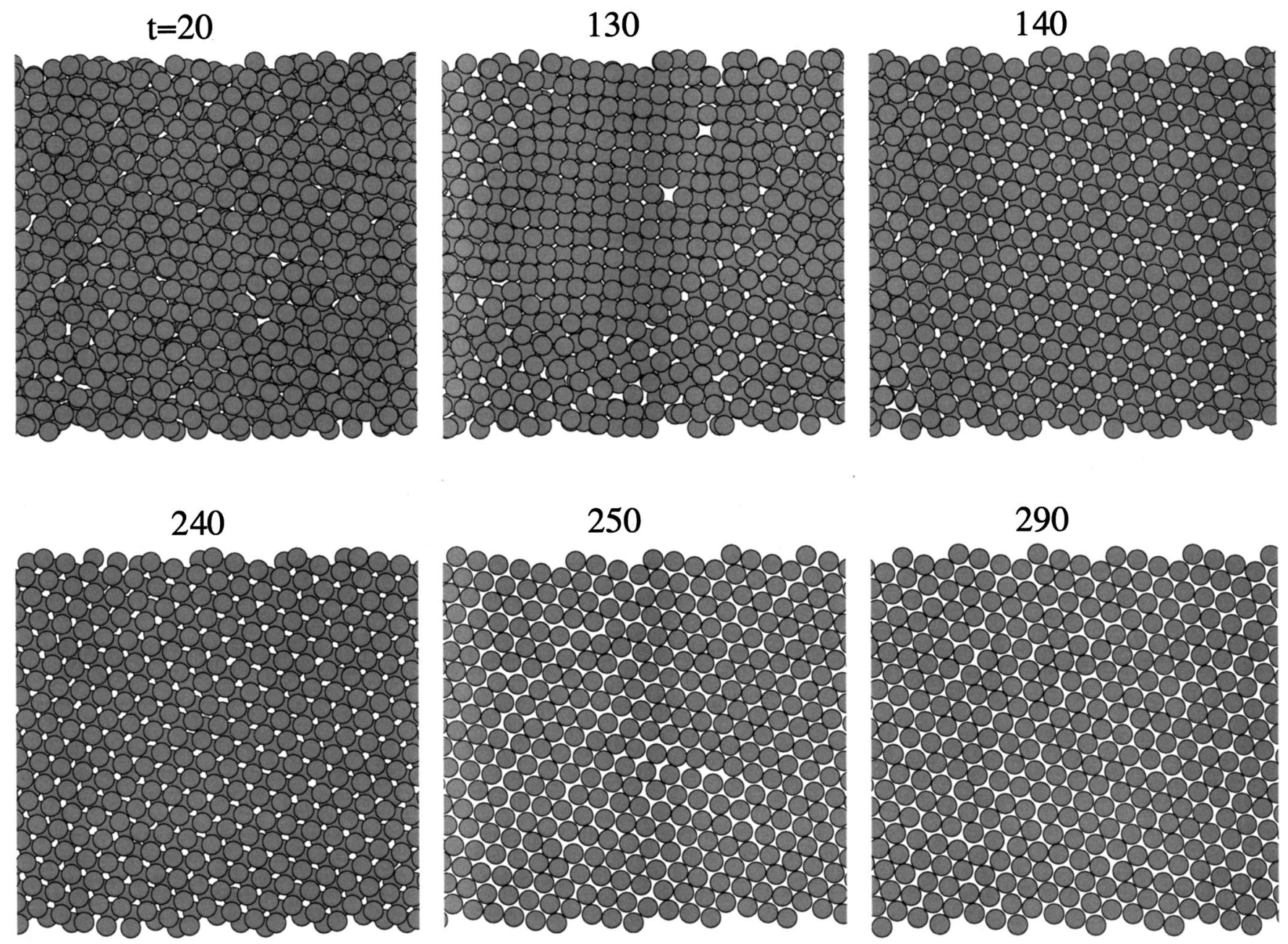

FIG. 16. Snapshot pictures of the lubricant layer during squeeze-out, after removing the block and substrate atoms. For model (A) and temperature $T$ $=300 \mathrm{~K}$.

transformed area by $b$ and assume that it had the same value in the original hexagonal structure (inspection of snapshots shows that this is a good approximation). The density of Xe atoms within the hexagonal and $\mathrm{fcc}(100)$ planes are $n_{1}$ $=2 /\left(b^{2} 3^{1 / 2}\right)$ and $n_{2}=1 / b^{2}$, respectively. The distance between the atomic planes in the two cases are $l_{1}=(2 / 3)^{1 / 2} b$ and $l_{2}=b / 2^{1 / 2}$, respectively. If we assume that there are $n \mathrm{Xe}$ layers parallel to the solid walls, then during the phase transformation the solid surfaces will approach each other by $\Delta z=(n-1)\left(l_{1}-l_{2}\right)$. This will result in a gain of elastic energy of $P A \Delta z$, where $A$ is the surface area which has undergone the transformation. During this transformation the number of adsorbates in contact with the solids walls will decrease by $\Delta N$ (these adsorbates are effectively transfered into the Xe reservoir outside the contact area). For $n>1$ we get $\Delta N=2\left(n_{1}-n_{2}\right) A$. The energy required for this removal process is approximately $\Delta N\left(E_{B}-2 \epsilon_{0}\right)$. Here $E_{B}$ is the Xe adsorption energy on a solid wall and $2 \epsilon_{0}$ is (approximately) the change in the $\mathrm{Xe}-\mathrm{Xe}$ interaction energy between an $\mathrm{Xe}$ atom in the liquid reservoir and when it is adsorbed on the solid surface in the interface region. This energy depends slightly on the exact number of layers $n$, but this is not important in the present case as $E_{B} \gg \epsilon_{0}$. Using the equation $P A \Delta z \approx \Delta N\left(E_{B}-2 \epsilon_{0}\right)$ we obtain

$$
P \approx 2^{3 / 2} \frac{E_{B}-2 \epsilon_{0}}{(n-1) b^{3}} .
$$

For $n=3$ this gives in the present case $P \approx 3 \mathrm{GPa}$ which is in excellent agreement with the observed pressure $(\sim 2.5 \mathrm{GPa})$ in the region where the transformation occurs for $n=3 .{ }^{11}$ Similarly, for $n=2$, Eq. (1) gives $P \approx 6 \mathrm{GPa}$ which again is in excellent agreement with the observed maximum pressure $(\sim 5 \mathrm{GPa})$ at the point where the transformation occurs for $n=2$.

Figure 17 shows snapshots of the lubrication film during the nucleation of the $n=2 \rightarrow 1$ squeeze-out. The light and dark particles denote the two different Xe monolayers. At $t$ $=243.6$, i.e., immediately before the onset of the squeezeout, a fcc(100)-layer region has been formed. In snapshot $t$ $=244.2$ the $n=2 \rightarrow 1$ transition has nucleated in the transformed region. The layering transition initially spreads rapidly by converting the fcc(100) layers into the more close- 

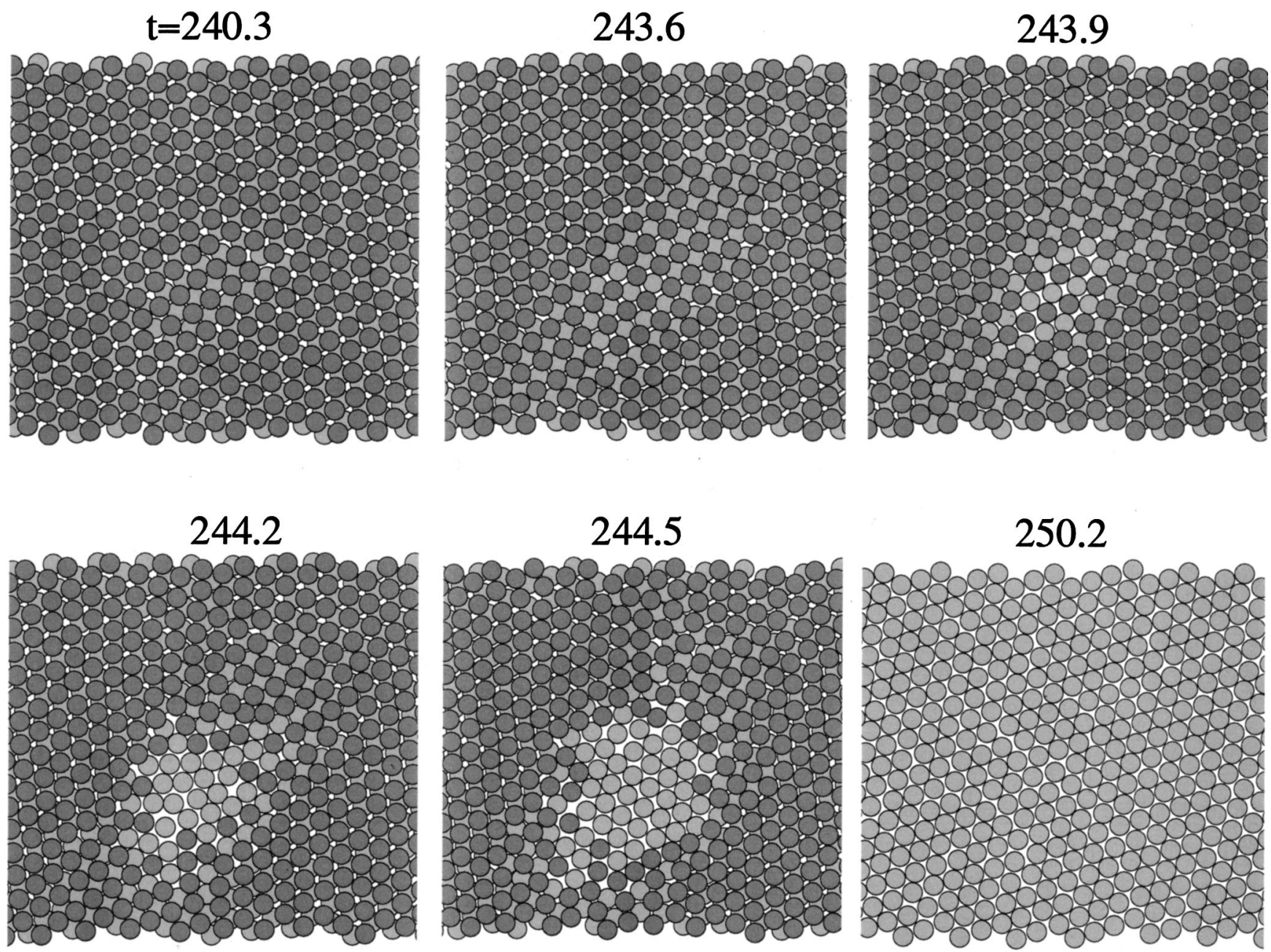

FIG. 17. Snapshot pictures of the lubricant layer for times close to the point where the $n=2 \rightarrow 1$ squeeze-out transition occurs. For model (A) and temperature $T=300 \mathrm{~K}$.

packed hexagonal layers. When this process is completed, the squeeze-out occurs by sliding the lubricant slabs relative to the solid surfaces, as described above.

Figure 18 shows a picture from an inclined view angle during the nucleation of the squeeze-out. At $t=243.9$ a small "hole" has just been formed in the $n=2$ layer (see Fig. 17), which then rapidly expands by converting the fcc(100) layers into the hexagonal ones.

\section{B. Incommensurate pinned layer}

We consider now case (B), where the Xe monolayer forms a pinned incommensurate state [see Fig. 10(a)]. The parameters relevant for this case were given in Sec. III. Note that for a single atom adsorbed on the substrate the binding energy is highest in the hollow sites $\left(E_{B}=0.46 \mathrm{eV}\right)$ and $13 \%$ smaller in the on-top sites, so that the lateral corrugation of the ground state potential energy surface in the present case equals $\Delta E=60 \mathrm{meV}$. The substrate lattice constant $a$ $=3.2 \AA$ is much smaller than the equilibrium $\mathrm{Xe}-\mathrm{Xe}$ separation (which is close to $r_{0}=4 \AA$ ), resulting in the observed incommensurate pinned structure. All the results presented in this section are for $T=200 \mathrm{~K}$.
Figure 19 (top) shows the average perpendicular stress (or pressure) acting on the substrate (or block) as a function of the displacement of the block towards the substrate. The block and the substrate are initially separated by about four Xe monolayers. Calculations are presented for the squeezing velocity $v_{z} \approx 4.4 \mathrm{~m} / \mathrm{s}$. The upper curve (a) is for zero sliding velocity $\left(v_{x}=0\right)$ while the lower curve (b) is for $v_{x}$ $\approx 17.7 \mathrm{~m} / \mathrm{s}$. For clarity, the latter curve is displaced towards negative pressure by $0.2 \mathrm{GPa}$. Note that, in contrast to the incommensurate case (A), the larger lateral atomic corrugation experienced by the lubrication atoms in the present case produces a much slower and sluggish squeeze-out, and only very weak bumps can be detected in the upper curve for the $n=4 \rightarrow 3$ and $n=3 \rightarrow 2$ transitions. (More well-defined bumps would occur at lower squeezing velocity $v_{z}$, but no such simulation was performed.) Note, however, that the layering transitions start at lower pressures in the present case than in case (A): this result is perhaps expected because the adsorbate-substrate binding energy (and hence the spreading pressure) is much smaller in this case than in case (A). Thus, while the lateral corrugation of the adsorbate-substrate interaction potential has a great influence on the squeeze-out velocity, it is much less important for the nucleation of the 

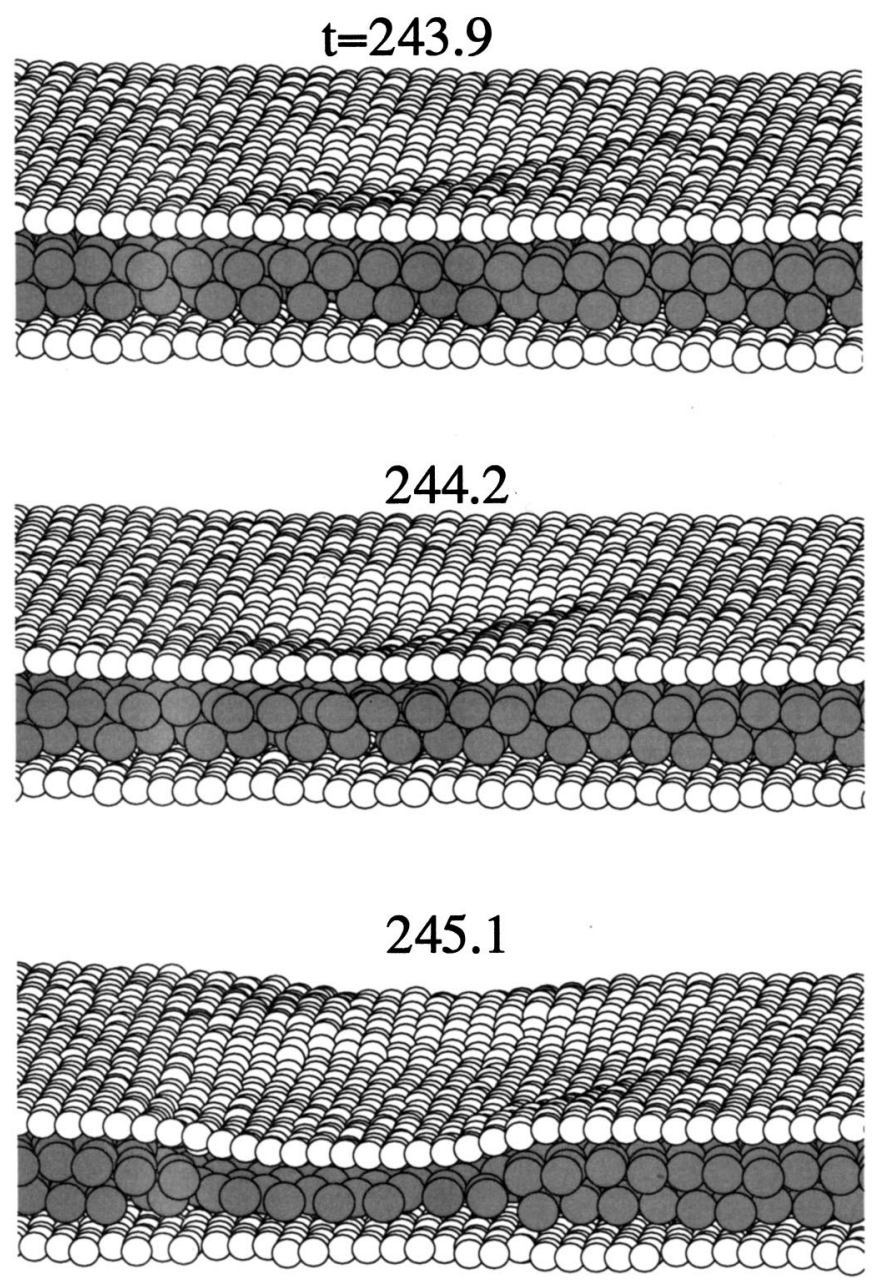

FIG. 18. Snapshot pictures, at an inclined view angle, of the central region of the system at three time points immediately after the $n=2 \rightarrow 1$ squeezeout transition has nucleated. For model (A) and temperature $T=300 \mathrm{~K}$.

layering transition, which depends mainly on the adsorbatesubstrate binding energy via the spreading pressure (see Sec. II). The bottom figure shows the average kinetic energy of a $\mathrm{Xe}$ atom. The upper curve (a) is for $v_{x}=0$, i.e., without lateral sliding, while the lower curve (b) is for $v_{x} \approx 17.7 \mathrm{~m} / \mathrm{s}$. For clarity, the latter curve is displaced towards lower energy by $30 \mathrm{~K}$. Note that in the present case, because of the slow and sluggish squeeze-out, the maximum kinetic energy picked up by the $\mathrm{Xe}$ atoms during squeeze out is much smaller than for the incommensurate case (A), where the lateral barrier experienced by the $\mathrm{Xe}$ atoms is much lower.

Figure 20 shows a sequence of snapshots of the central interface region during squeezing. The upper surface of the block moves towards the substrate with the velocity $v_{z}$ $\approx 4.4 \mathrm{~m} / \mathrm{s}$. Note that in the last snapshot picture a trapped $n$ $=2$ island occurs, surrounded by a single Xe monolayer.

Figure 21 shows snapshot pictures of the central part of the lubrication layer for the case shown in Fig. 20. At $t$ $=180$, a $n=3$ layer occurs and, except for a line defect, within each layer the $\mathrm{Xe}$ atoms form a nearly perfect hexagonal structure. Note that at $t=200$, i.e., immediately before the onset of the $n=3 \rightarrow 2$ layering transition, the central region has changed structure from hexagonal to fcc(100) lay-
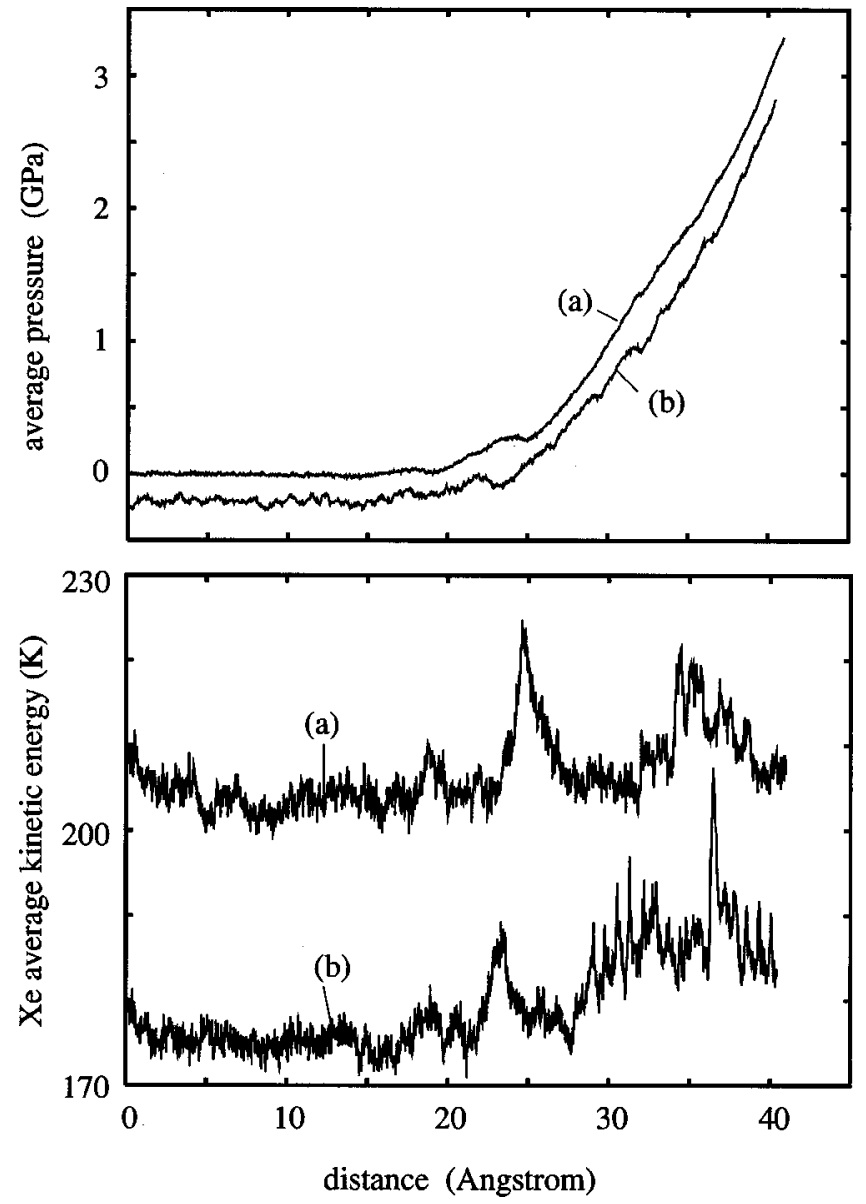

FIG. 19. The dependence of the average pressure (top) and the average Xe kinetic energy (bottom) on the distance the upper surface of the block has moved towards the bottom surface of the substrate. Results are shown for (a) squeezing with $v_{z} \approx 4.4 \mathrm{~m} / \mathrm{s}$ and (b) squeezing $\left(v_{z} \approx 4.4 \mathrm{~m} / \mathrm{s}\right)$ and sliding at $v_{x}=17.7 \mathrm{~m} / \mathrm{s}$. For model (B) with $T=200 \mathrm{~K}$.

ers: as discussed earlier, this allows the solid surfaces to approach each other, and results in a gain of elastic energy, partially compensated by the loss of adsorbate-substrate binding energy. As before, the more open structure of the fcc(100) layers also facilitates the transition $n=3 \rightarrow 2$ as it allows for greater in-plane density fluctuations. Thus the transition $n=3 \rightarrow 2$ occurs shortly after the transformation of the Xe film into the $\mathrm{fcc}(100)$ layer structure. Immediately after the $n=3 \rightarrow 2$ transition, a nearly perfect hexagonal Xe bilayer is formed. The sequence of transformations is repeated at higher pressures: immediately before the onset of the transition $n=2 \rightarrow 1$, the lubrication film in the central region of the contact area again switches to the fcc(100) layer structure, facilitating density fluctuations and the nucleation of a hole. A complete squeeze-out of the second monolayer does not occur in the present case but, as mentioned above, an $n=2$ island is trapped, completely surrounded by $n=1$ film area. The size and structure of the trapped island are illustrated by Fig. 22.

We have also studied the system during squeezing and sliding [see curve (b) in Fig. 19]. The upper surface of the block moves towards the bottom surface of the substrate with the velocity $v_{z} \approx 4.4 \mathrm{~m} / \mathrm{s}$ and parallel to the substrate with four times higher velocity $\left(v_{x} \approx 17.7 \mathrm{~m} / \mathrm{s}\right)$. In this case we 


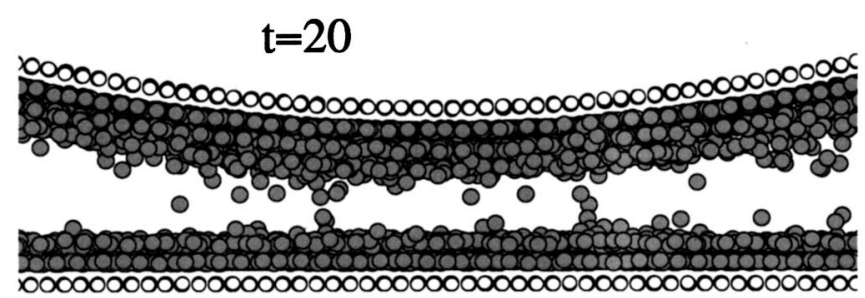

60

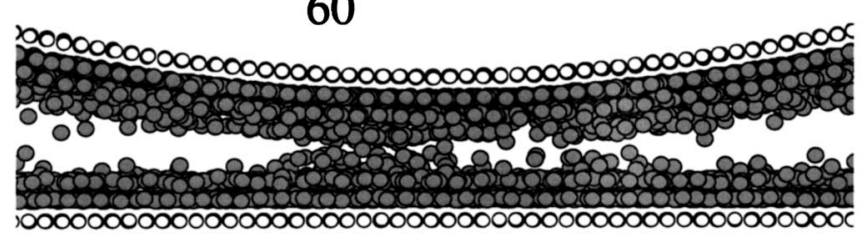

100

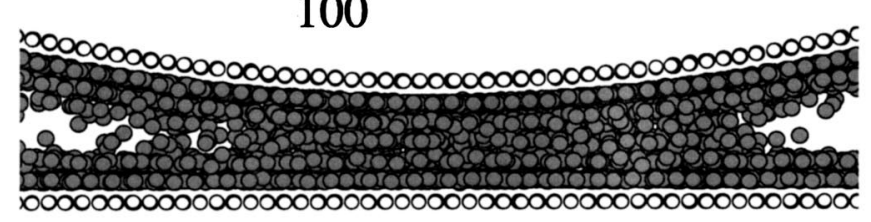

140

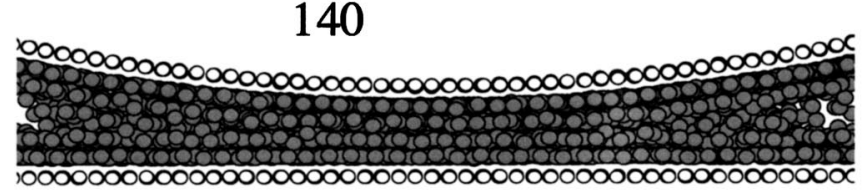

180

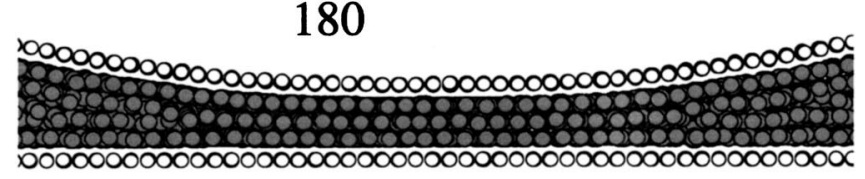

200

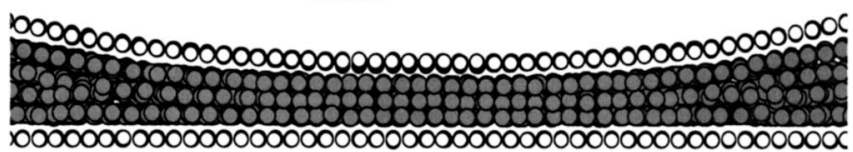

220

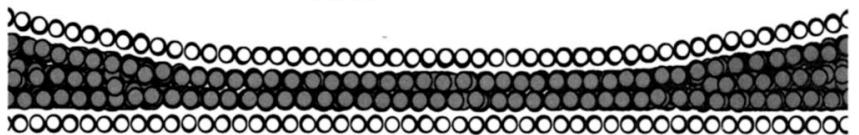

240

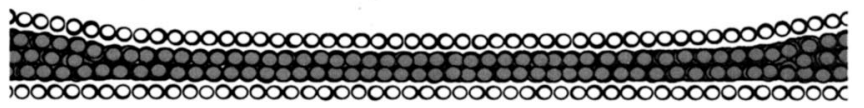

260
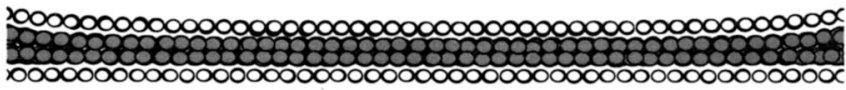

280

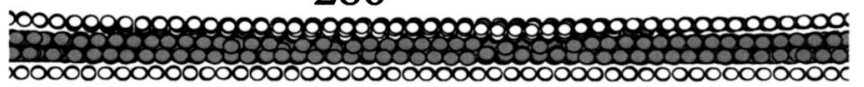

300

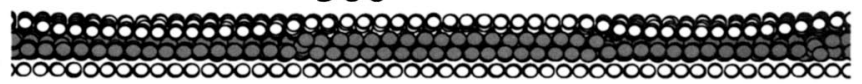

360

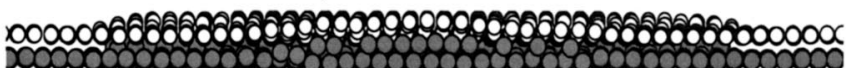
0000000000000000000000000000000000000000000

FIG. 20. Snapshot pictures during squeeze-out. The time of each snapshot is indicated. Note that a trapped $n=2$ island occurs in the last snapshot picture. For model (B) at $T=200 \mathrm{~K}$.

find that the final state of the lubrication film consists of a single Xe monolayer in the close contact region, in contrast to the $v_{x}=0$ case, where a trapped $n=2$ island remains under otherwise identical squeezing conditions. This is illustrated in Fig. 23, which shows snapshot pictures of the central part of the lubrication layer during squeezing and sliding. At $t=220$ a bilayer occurs and within each layer the Xe atoms form nearly perfect hexagonal structures. At $t=240$, immediately before the onset of the $n=2 \rightarrow 1$ squeeze-out, the central region has changed to the fcc(100)-layer structure which facilitates the $n=2 \rightarrow 1$ transition. Thus the layering transition $n=2 \rightarrow 1$ occurs rapidly after the transformation of the Xe-film into the (100)fcc layer structure. After the transition $n=2 \rightarrow 1$ a nearly perfect hexagonal Xe monolayer is formed. Stable sliding with a single monolayer lubricant film is observed for a wide range of applied pressures. However, this state is unstable during sliding at high pressure, and, after a short time, first a domain wall superstructure is formed (the snapshot at $t=340$ shows an example of this structure, displaying also apparent structural defects) and, with continuing sliding (at high velocity) at constant pressure (i.e., with $v_{z}=0$ ), this monolayer gets more and more diluted, until it vanishes after a sufficiently long time.

\section{Commensurate layer}

We consider now case (C), where the lubricant monolayer forms a commensurate $(1 \times 1)$ structure. The parameters relevant for this case were given in Sec. III, and the equilibrium structure in the absence of an applied shear force is shown in Fig. 10(b). Note that for a single atom adsorbed on the substrate the binding energy is highest in the hollow sites $\left(E_{B}=0.31 \mathrm{eV}\right)$ and $39 \%$ smaller in the on-top sites, so that the lateral corrugation of the ground state potential energy surface in the present case equals $\Delta E=0.12 \mathrm{eV}$. The substrate lattice constant $a=4.4 \AA$ is close to the natural $\mathrm{Xe}-\mathrm{Xe}$ separation (which is close to $r_{0}=4 \AA$ ). All the results presented below are for $T=80 \mathrm{~K}$.

Figure 24 (top) shows the average perpendicular stress acting on the substrate (or block) as a function of the displacement of the block towards the substrate. The block and the substrate are initially separated by about four Xe monolayers. Calculations are presented for the squeeze velocity $v_{z} \approx 4.6 \mathrm{~m} / \mathrm{s}$. The upper curve (a) is without lateral sliding $\left(v_{x}=0\right)$ while the lower curve (b) is for $v_{x}=18.3 \mathrm{~m} / \mathrm{s}$. Note that commensurate $(1 \times 1)$ adsorbate layers are strongly pinned, and even though in the present case the Xe substrate 

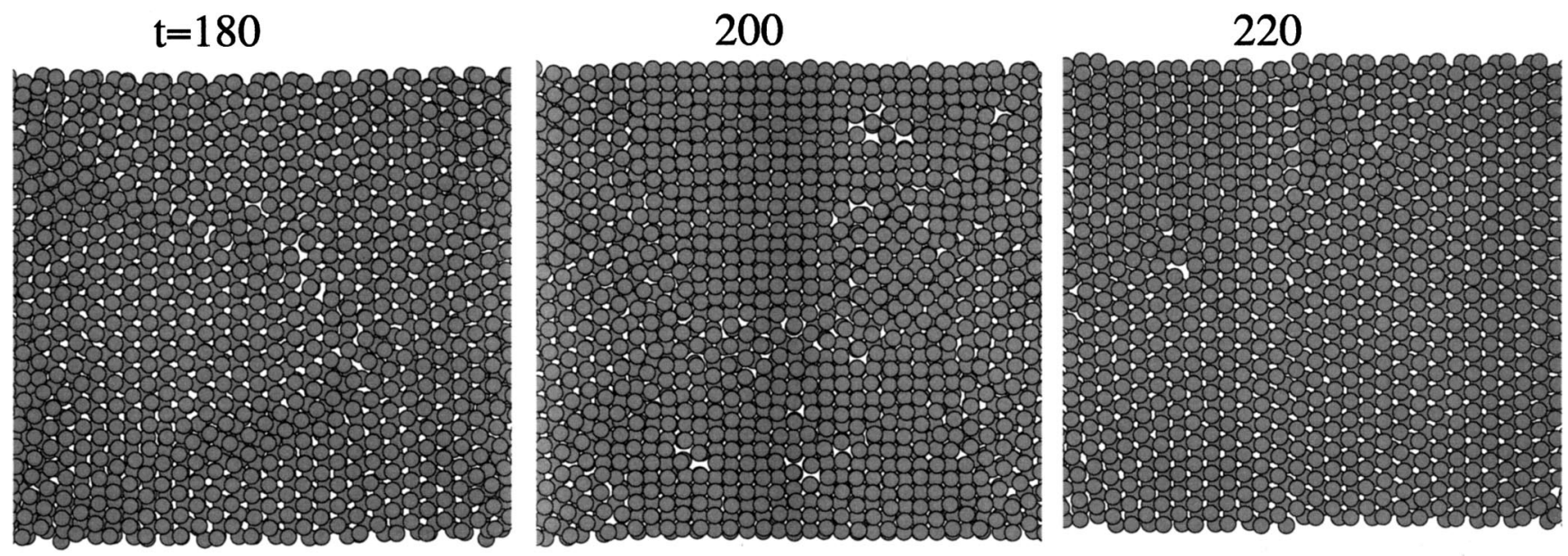

260

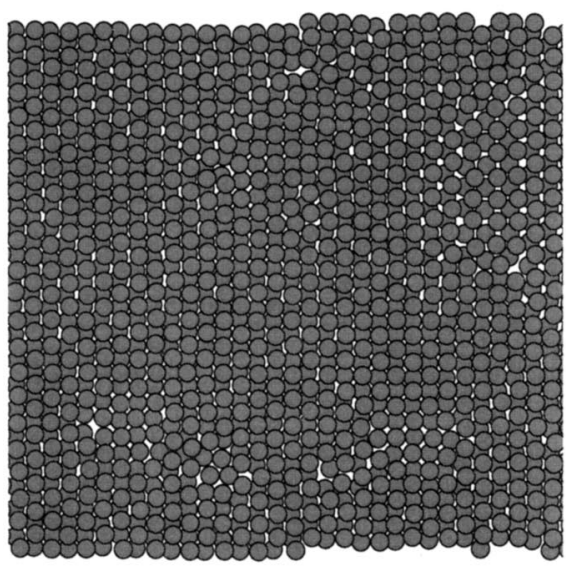

280

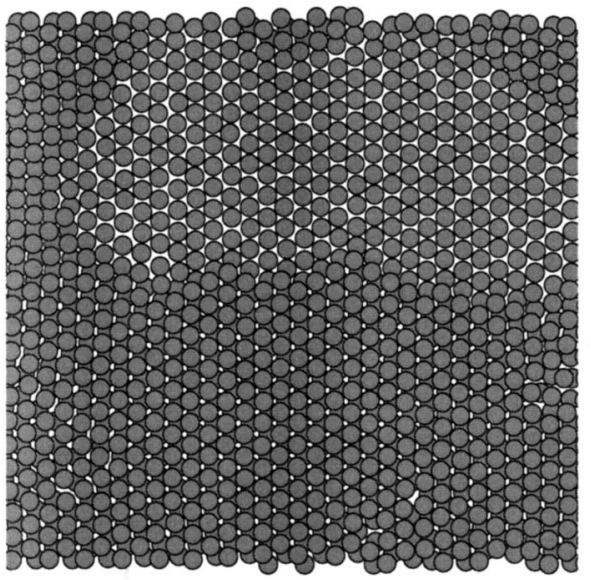

360

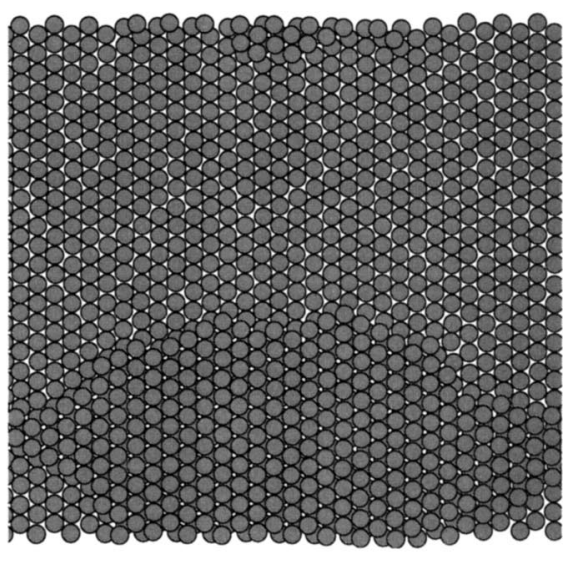

FIG. 21. Snapshot pictures of the lubricant layer during squeeze-out, after removing the block and substrate atoms. Note the $n=2$ island in the last snapshot picture. For model (B) and temperature $T=200 \mathrm{~K}$.

binding energy is much smaller than for the incommensurate case (A), it is very hard to squeeze out the lubrication film when no lateral sliding is present. As already observed in the previous case, lateral sliding tends to break the pinning by turning the adsorbate layer into a fluidized or disordered state, thus facilitating the squeeze-out of the lubricant (see Fig. 26). However, the squeeze out is very slow and sluggish, and, in this case, it is not possible to detect any structure in the pressure vs. distance curves [the small oscillations in curve (b) reflect atomistic stick-slip motion of the lubrication film]. The bottom figure shows the average kinetic en-

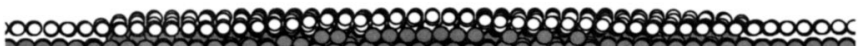


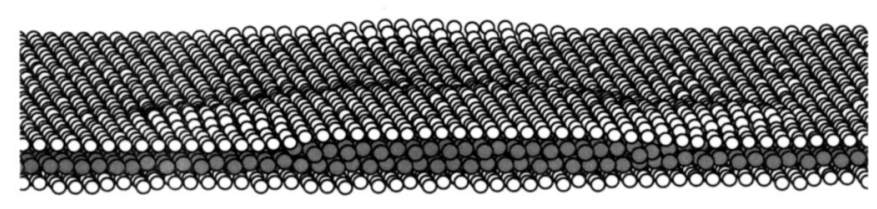

FIG. 22. Snapshot pictures from two different view angles, of the trapped island at the last time point in Fig. 21. For model (B) at $T=200 \mathrm{~K}$. ergy of a Xe atom. The lower curve (a) is without lateral sliding $\left(v_{x}=0\right)$ while the upper curve (b) is for $v_{x}$ $\approx 18.3 \mathrm{~m} / \mathrm{s}$. Note that the kinetic energy picked up by the Xe atoms during squeeze-out is much smaller than for the incommensurate case (A), where the lateral barrier experienced by the Xe atoms is much smaller. Note also the presence of stick-slip oscillations in the Xe kinetic energy during sliding.

Figure 25 shows the (average) frictional shear stress acting on the block during sliding [case (b)], which displays clear stick-slip oscillations. Since the average pressure at the end of the simulation is about $\sim 3 \mathrm{GPa}$ and the average shear stress $\sim 0.3 \mathrm{GPa}$ (see figure), the kinetic friction coefficient in the present case is of order $\sim 0.1$, which is typical for boundary lubricated surfaces.

Figure 26 shows a sequence of snapshot pictures of the central region during squeezing and sliding. Note that the last configuration has a single monolayer of lubrication atoms in the central contact region, in contrast to the case where $v_{x}=0$, where it was not possible to squeeze out any lubrication atom. Figure 27 shows snapshot pictures of the central part of the lubrication layer for the system in Fig. 26. In general, the lubrication film forms commensurate $(1 \times 1)$ layers, but with many defects and incomplete layers. Thus 

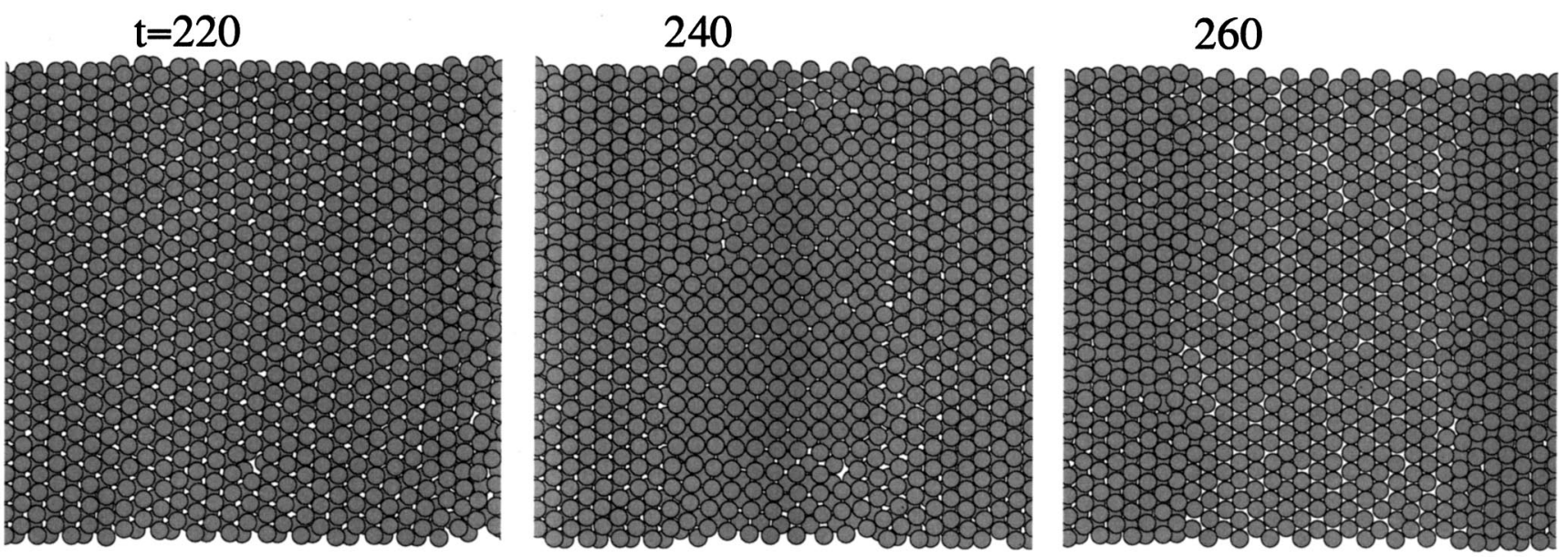

280

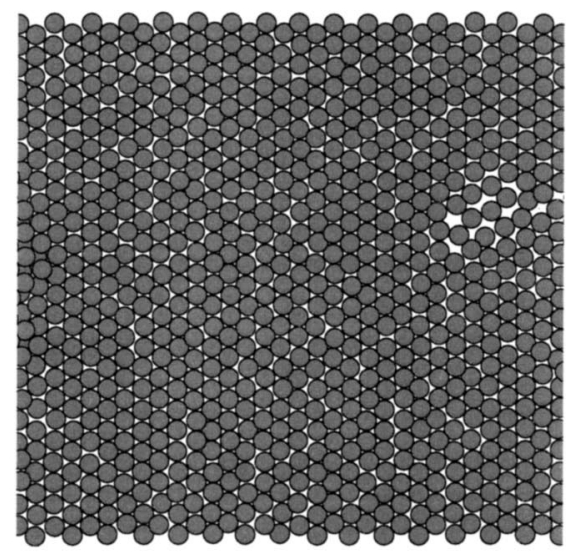

320

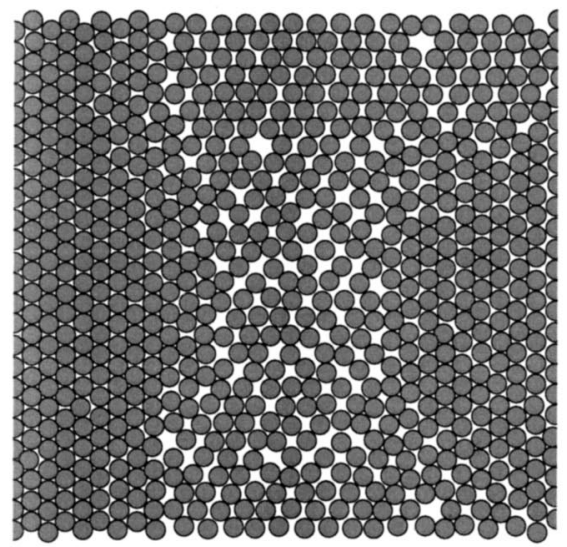

340

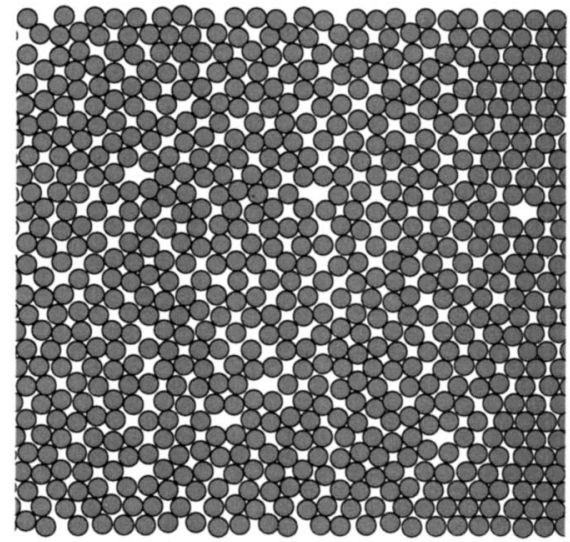

FIG. 23. Snapshot pictures of the lubricant layer during squeeze-out and sliding, after removing the block and substrate atoms. For model (B) and temperature $T=200 \mathrm{~K}$.

the picture at $t=160$ has a small region with $n=1$ at the center of the $n=2$ film. The squeeze out of the second layer occurs very slowly, and in a complex manner.

Figure 28 reports the $t=230$ snapshot in Fig. 27 with graphical changes that highlight the relation between the structure of the lubricant and of the solid surfaces: the radius of the $\mathrm{Xe}$ atoms has been reduced, and the top layer of substrate atoms and bottom layer of block atoms have been included (open circles) in this illustration. A detailed analysis shows that the $\mathrm{Xe}$ monolayer region above symbol $\mathrm{A}$ is pinned to the block and moves with the speed of the block to the left. The bilayer region below symbol B is pinned to the substrate and hence stationary. At the boundary between these two regions local disordering or fluidization occurs, contributing to the energy dissipation and to the friction force.

\section{SUMMARY AND CONCLUSION}

We have presented the results of computer simulations where, for the first time, both long-range elasticity and curved surfaces have been included in a realistic manner. The most important results of the study are as follows: (i) Atomic fluids confined to narrow spaces between flat solid surfaces form well-defined layers parallel to the surfaces. We expect this result to be valid for molecular lubricants as well. As a function of the external pressure, we observe discrete layering transitions $(n \rightarrow n-1)$, which are thermally activated. If the lubrication layers are unpinned or weakly pinned by the solid walls, the squeeze-out of a layer can occur rapidly. For pinned incommensurate or commensurate layers, the squeeze-out can be slow and sluggish.

(ii) Sometimes we observe incomplete squeeze-out, resulting in trapped islands, e.g., $n=2$ island completely surrounded by $n=1$ film area. This has also been observed in recent experiments. ${ }^{9}$

(iii) For pinned lubrication films, squeeze-out occurs much more easily when lateral sliding is superimposed to squeezing. This results from the breaking up of the pinned structures induced by sliding. Also this effect has been observed in experiments. ${ }^{3}$

(iv) It is very difficult, if possible at all, to squeeze out a commensurate solid lubrication film in the absence of lateral sliding. However, when the two surfaces slide with respect to each other, the lubricant film can often be completely squeezed out of the interface. 

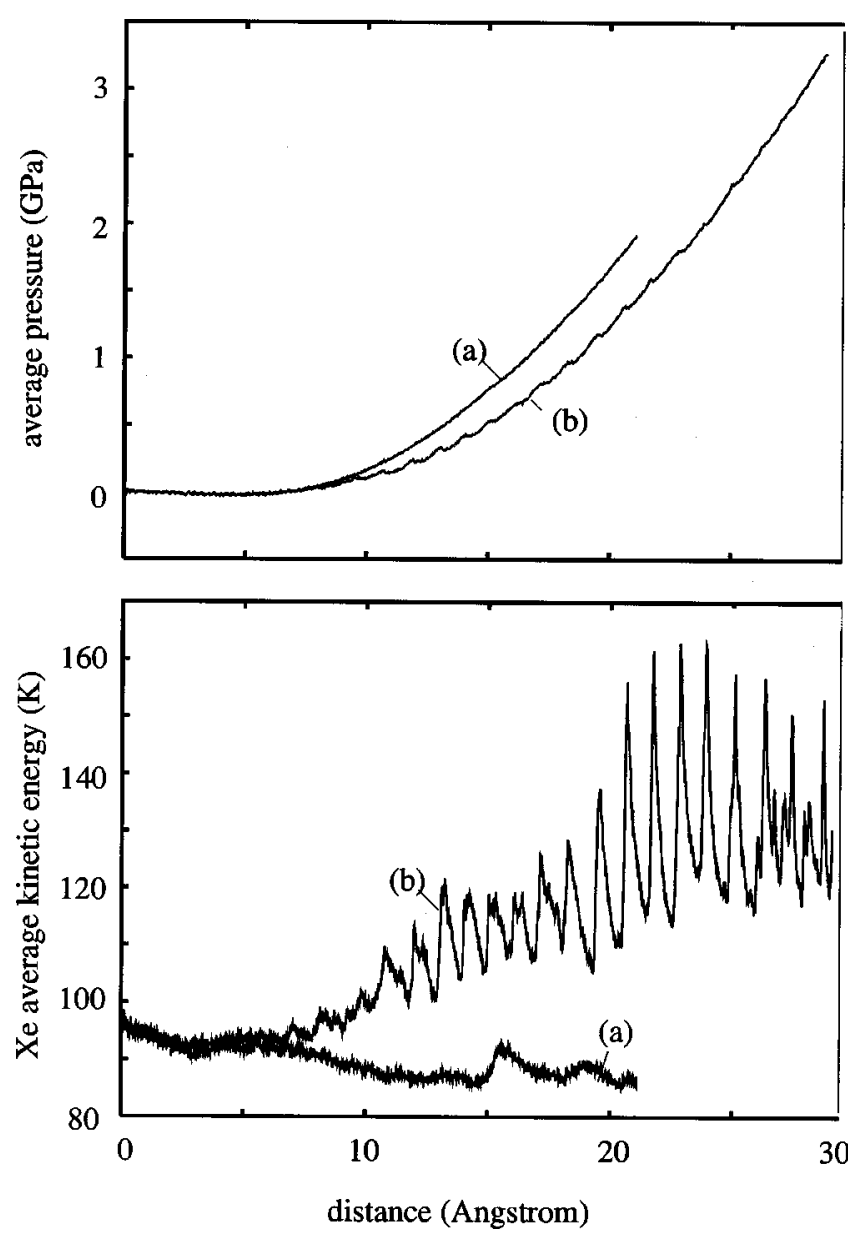

FIG. 24. The dependence of the average pressure (top) and the average Xe kinetic energy (bottom) on the distance the upper surface of the block has moved towards the bottom surface of the substrate. Results are shown for (a) squeezing with $v_{z} \approx 4.6 \mathrm{~m} / \mathrm{s}$ and (b) squeezing $\left(v_{z} \approx 4.6 \mathrm{~m} / \mathrm{s}\right)$ and sliding at $v_{x}=18.3 \mathrm{~m} / \mathrm{s}$. For model (C) with $T=80 \mathrm{~K}$.

(v) In some cases we have observed, immediately before each layering transition, the formation of an intermediate phase in the lubrication film, localized in the high pressure region between the solids. This transformation allows the

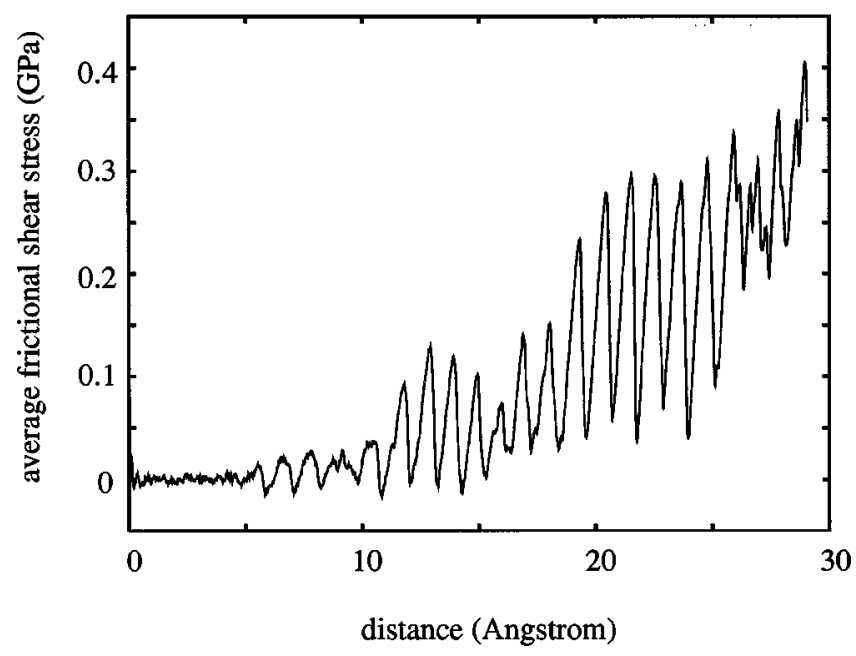

FIG. 25. The average interfacial shear stress acting on the block during sliding and squeezing during simulation (b) in Fig. 24. surfaces to approach each other, resulting in a gain of elastic energy, which is the driving force for the layering transformation.

(vi) When the lateral barrier associated with the sliding of a lubricant layer over the solid surfaces is small compared with the effective barrier experienced when the lubricant layers slide relative to each other, no slip occurs between the lubricant layers during squeeze-out, but slip occurs only at the solid-lubricant interfaces. That is, the lubricant layer slides as a single unit relative to the solid walls. This is observed for incommensurate layers which are unpinned or weakly pinned by the substrate, while it is not observed for strongly pinned commensurate layers, where the squeeze out (which now only occur during sliding) is much more complex.

We are at present extending the work presented above to sliding at constant external load (or pressure). ${ }^{4}$ We will also consider other lubricants, e.g., chain molecules, as well as solids with different lattice constant and different elastic properties (e.g., soft elastic solids such as rubber). We plan also to study the influence of different types of surface corrugation on squeeze-out and sliding dynamics.

\section{ACKNOWLEDGMENTS}

B.N.J.P acknowledges support by a BMBF grant related to the German-Israeli Project Cooperation "Novel Tribological Strategies from the Nano- to Meso-Scales." He also thanks F. Mugele and M. Salmeron for interesting discussions and for a preprint.

\section{APPENDIX}

In this appendix we describe the model we have used in the computer simulations. The coordinate vector of the particle associated to lattice site $\mathbf{n}=\left(n_{x}, n_{y}\right) \quad\left(n_{x}=1, . ., N_{x}\right.$; $\left.n_{y}=1, . ., N_{y}\right)$ in the bottom layer of atoms in the block is denoted by $\mathbf{r}_{\mathbf{n}}$, and the coordinate vector of the corresponding particle in the top layer of the substrate is denoted by $\mathbf{r}_{\mathbf{n}}^{*}$ where $\mathbf{n}=\left(n_{x}, n_{y}\right) \quad\left(n_{x}=1, . ., N_{x}^{*} ; n_{y}=1, . ., N_{y}^{*}\right)$ with $L_{x}=N_{x} a=N_{x}^{*} a^{*}$ and $L_{y}=N_{y} a=N_{y}^{*} a^{*}$, where $a$ and $a^{*}$ are the lattice constants of the block and substrate, respectively, and $L_{x}$ and $L_{y}$ the width in the $x$ - and $y$-directions of the system (we use periodic boundary conditions in the $x, y$-directions). Between the block and substrate we will assume a layer (monolayer or more) of lubrication atoms, which interact via Lenard-Jones pair potentials. We also assume that the lubrication molecules interact with the atoms of the solid surfaces via Lenard-Jones potentials (see below).

In this article we assume that the block has a cosine corrugation. In this case, when the block is pushed towards the substrate, the area of real contact will depend on the external load. With a corrugated surface we can study the transition from hydrodynamic lubrication to boundary lubrication, and also how the layering transitions occur as monolayers of lubrication "fluids" are squeezed out from the area of real contact between the block and the substrate. All quantities referring to the substrate will be distinguished from those of the block by the upper index *. Associated with the block we introduce the matrices 


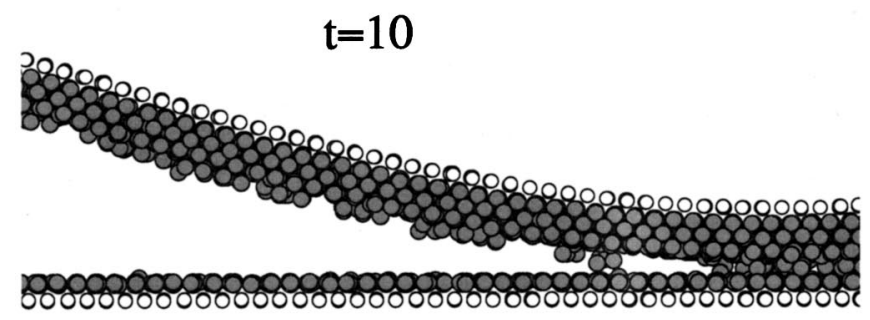

60

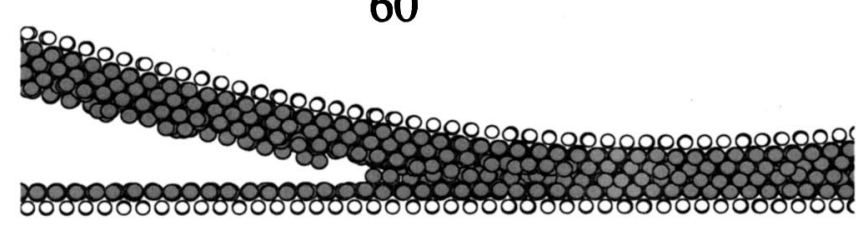

90

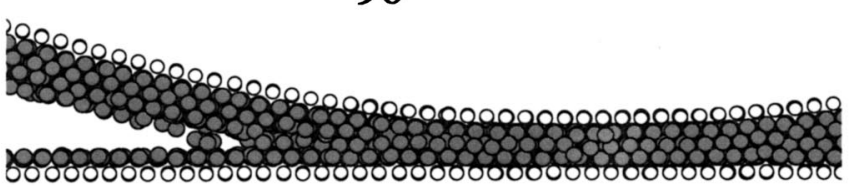

110

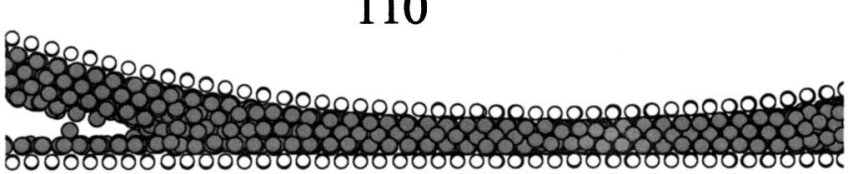

150

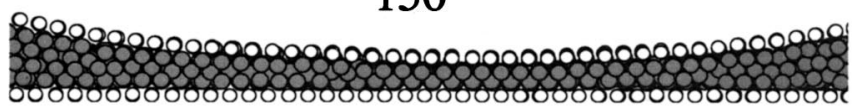

160

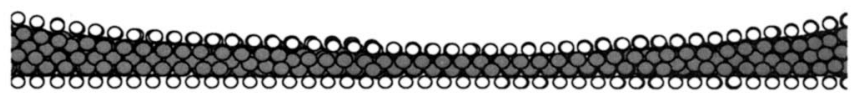

190

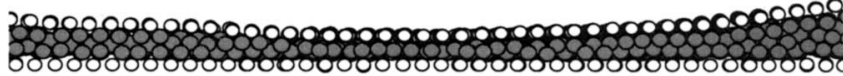

210

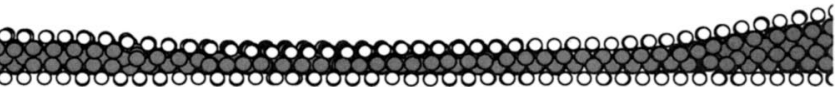

230

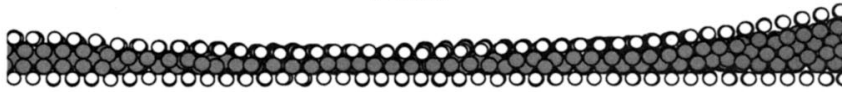

FIG. 26. Snapshot pictures during squeeze-out and sliding [case (b) in Fig. 24]. The time of each snapshot is indicated. For model (C) at $T=80 \mathrm{~K}$.

$$
\begin{aligned}
K_{x} & =\left(\begin{array}{ccc}
k_{0} & 0 & 0 \\
0 & k_{0 B} & 0 \\
0 & 0 & k_{0 B}
\end{array}\right), K_{y}=\left(\begin{array}{ccc}
k_{0 B} & 0 & 0 \\
0 & k_{0} & 0 \\
0 & 0 & k_{0 B}
\end{array}\right), \\
K_{1} & =\left(\begin{array}{ccc}
k_{1 B} & 0 & 0 \\
0 & k_{1 B} & 0 \\
0 & 0 & k_{1}
\end{array}\right),
\end{aligned}
$$

and similar matrices $K_{x}^{*}, K_{y}^{*}$, and $K_{1}^{*}$ for the substrate. The quantities $k_{0}, k_{0 B}, k_{1}$, and $k_{1 B}$ were defined in Sec. III.

\section{Equations of motion}

The equation of motion for the center-of-mass $\mathbf{X}(t)$ $=(X, Y, Z)(t)$ of the block is

$$
\begin{aligned}
M \ddot{\mathbf{X}}= & k_{s}\left[X_{s}(t)-X\right] \hat{x}+m \eta \sum_{\mathbf{n}}\left(\dot{\mathbf{r}}_{\mathbf{n}}-\dot{\mathbf{X}}\right)-\sum_{\mathbf{n}} \mathbf{f}_{\mathbf{n}} \\
& -K_{1} \cdot \sum_{\mathbf{n}}\left[\mathbf{X}+\mathbf{n} a+h_{\mathbf{n}} \hat{z}-\mathbf{r}_{\mathbf{n}}-W \hat{z}\right],
\end{aligned}
$$

where $X_{s}(t)$ is the coordinate for the free end of the spring, which, in most cases, is assumed to be $X_{s}=v_{s} t$, with a constant spring velocity $v_{s}$.

The equations of motion for the atoms in the bottom layer of the upper block are

$$
\begin{aligned}
m \ddot{\mathbf{r}}_{\mathbf{n}}= & -m \eta\left(\dot{\mathbf{r}}_{\mathbf{n}}-\dot{\mathbf{X}}\right)+K_{x} \cdot\left(\mathbf{r}_{\mathbf{n}+\hat{x}}+\mathbf{r}_{\mathbf{n}-\hat{x}}-2 \mathbf{r}_{\mathbf{n}}\right) \\
& +K_{y} \cdot\left(\mathbf{r}_{\mathbf{n}+\hat{y}}+\mathbf{r}_{\mathbf{n}-\hat{y}}-2 \mathbf{r}_{\mathbf{n}}\right) \\
& +K_{1} \cdot\left[\mathbf{X}+\mathbf{n} a+h_{\mathbf{n}} \hat{z}-\mathbf{r}_{\mathbf{n}}-W \hat{z}\right]+\mathbf{F}_{\mathbf{n}}+\mathbf{f}_{\mathbf{n}},
\end{aligned}
$$

where

$$
\mathbf{F}_{\mathbf{n}}=-\frac{\partial V}{\partial \mathbf{r}_{\mathbf{n}}} .
$$

In our case, we have

$$
\begin{aligned}
V= & \sum_{\mathbf{n} i} v_{13}\left(\mathbf{r}_{\mathbf{n}}-\mathbf{x}_{i}\right)+\sum_{\mathbf{n} i} v_{12}\left(\mathbf{r}_{\mathbf{n}}^{*}-\mathbf{x}_{i}\right)+\sum_{\mathbf{n n}^{\prime}} v_{23}\left(\mathbf{r}_{\mathbf{n}}^{*}-\mathbf{r}_{\mathbf{n}^{\prime}}\right) \\
& +\frac{1}{2} \sum_{i j} v_{1}\left(\mathbf{x}_{i}-\mathbf{x}_{j}\right),
\end{aligned}
$$

where (with $r=|\mathbf{x}|$ )

$$
v_{1}(\mathbf{x})=4 \epsilon_{0}\left[\left(\frac{r_{0}}{r}\right)^{12}-\left(\frac{r_{0}}{r}\right)^{6}\right],
$$

and similar expressions are assumed for $v_{12}$ and $v_{13}$. In the present calculations we have neglected the direct interactions between the two solids, i.e., $v_{23}=0$. The "corrugation" function $h_{\mathbf{n}}$ is in principle arbitrary, but in this article we use

$$
h_{\mathbf{n}}=z_{0}-h_{0} \cos \left(q n_{x} a\right),
$$



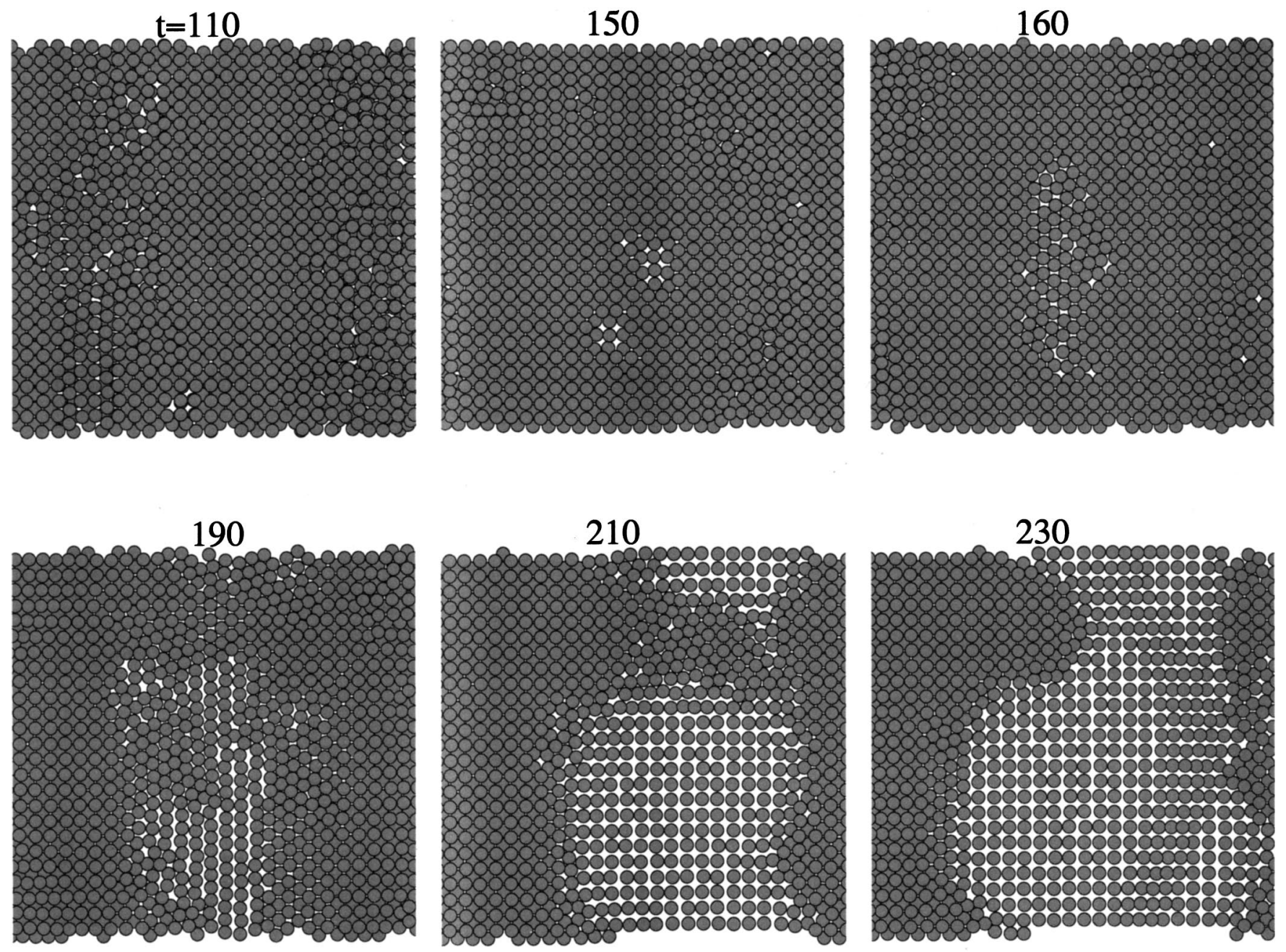

FIG. 27. Snapshot pictures of the lubricant layer during squeeze-out and sliding [case (b) in Fig. 24], after removing the block and substrate atoms. For model (C) and temperature $T=80 \mathrm{~K}$.

where $q=2 \pi / L_{x}$, where $L_{x}=N_{x} a$ is the width (or wavelength) in the $x$-direction of the surface profile of the block. In the present calculations we use $h_{0}=0.1 L_{x}$. Note that from Eqs. (A1) and (A2)

$$
M \ddot{\mathbf{X}}+\sum_{\mathbf{n}} m \ddot{\mathbf{r}}_{\mathbf{n}}=k_{s}\left[X_{s}(t)-X\right] \hat{x}+\sum_{\mathbf{n}} \mathbf{F}_{\mathbf{n}},
$$

i.e., the acceleration of the center-of-mass of the block is, as expected, determined by total external force acting on the block.

The force $\mathbf{f}_{\mathbf{n}}$ is a stochastically fluctuating force which is assumed to satisfy

$$
\left\langle f_{\mathbf{n}}^{i}(t) f_{\mathbf{n}^{\prime}}^{j}\left(t^{\prime}\right)\right\rangle=2 m \eta k_{B} T \delta\left(t-t^{\prime}\right) \delta_{i j} \delta_{\mathbf{n n}^{\prime}},
$$

where $T$ is the temperature.

The damping $\eta$ corresponds to the phonon energy radiated into the block and we show below that $\eta=A c_{T} / a$, where $c_{T}$ is the transverse sound velocity and $A$ a constant of order unity. The coordinate $z_{0}$ determines the (average) perpendicular pressure

$$
P=\sum_{\mathbf{n}} \hat{z} \cdot \mathbf{F}_{\mathbf{n}} /\left(N a^{2}\right),
$$

where $N=N_{x} N_{y}$.

The equations of motion for the lubrication atoms are

$$
m_{1} \ddot{\mathbf{x}}_{i}=-\frac{\partial V}{\partial \mathbf{x}_{i}} .
$$

The equation of motion for the top layer of atoms of the substrate

$$
\begin{aligned}
m^{*} \ddot{\mathbf{r}}_{\mathbf{n}}^{*} & +m^{*} \eta^{*} \dot{\mathbf{r}}_{\mathbf{n}}^{*} \\
= & K_{x}^{*} \cdot\left(\mathbf{r}_{\mathbf{n}+\hat{x}}^{*}+\mathbf{r}_{\mathbf{n}-\hat{x}}^{*}-2 \mathbf{r}_{\mathbf{n}}^{*}\right)+K_{y}^{*} \cdot\left(\mathbf{r}_{\mathbf{n}+\hat{y}}^{*}+\mathbf{r}_{\mathbf{n}-\hat{y}}^{*}-2 \mathbf{r}_{\mathbf{n}}^{*}\right) \\
& +K_{1}^{*} \cdot\left[h_{\mathbf{n}}^{*} \hat{z}+\mathbf{n} a^{*}-\mathbf{r}_{\mathbf{n}}^{*}-W^{*} \hat{z}\right]+\mathbf{F}_{\mathbf{n}}^{*}+\mathbf{f}_{\mathbf{n}}^{*},
\end{aligned}
$$

where

$$
\mathbf{F}_{\mathbf{n}}^{*}=-\frac{\partial V}{\partial \mathbf{r}_{\mathbf{n}}^{*}} .
$$

The force $\mathbf{f}_{\mathbf{n}}^{*}$ is a stochastically fluctuating force which is assumed to satisfy

$$
\left\langle f_{\mathbf{n}}^{* i}(t) f_{\mathbf{n}^{\prime}}^{* j}\left(t^{\prime}\right)\right\rangle=2 m^{*} \eta^{*} k_{B} T \delta\left(t-t^{\prime}\right) \delta_{i j} \delta_{\mathbf{n n}^{\prime}} .
$$




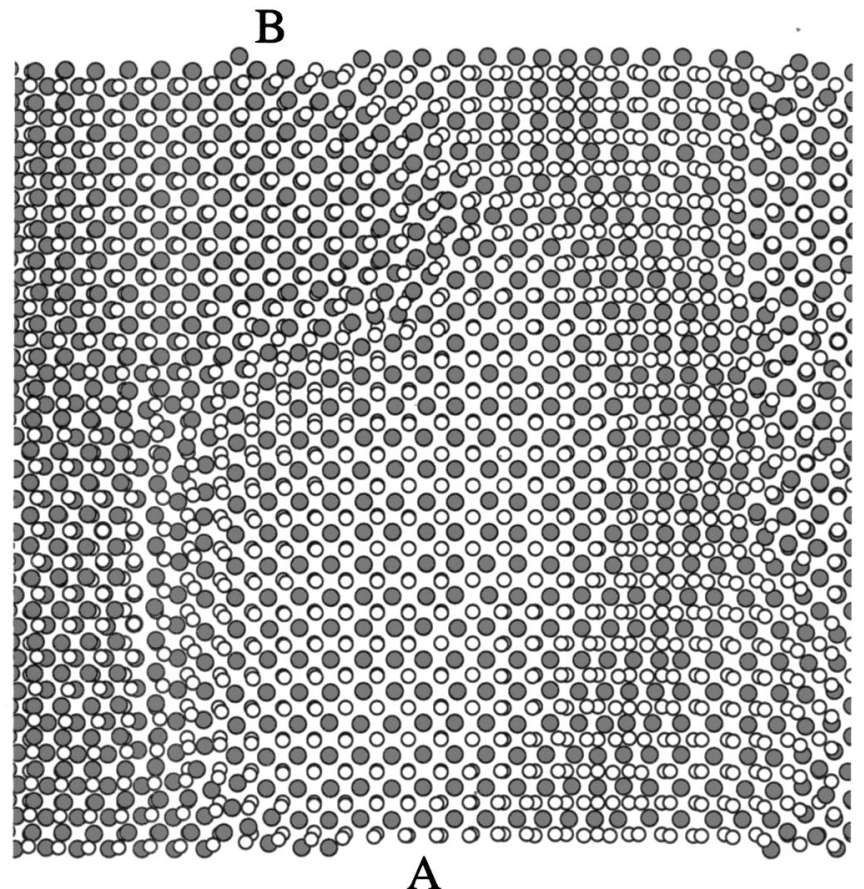

A

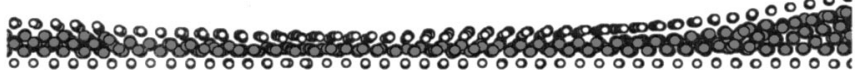

FIG. 28. Snapshot $t=230$ from Fig. 27 with different size of the lubrication atoms and with the substrate and block atoms included. The monolayer film region above $\mathrm{A}$ is pinned to the block and moves with the sliding velocity of the block. The bilayer below B is pinned to the substrate and hence stationary. For model (C) and temperature $T=80 \mathrm{~K}$.

\section{Dimensionless units}

It is convenient to measure time in units of $\tau$ $=\left(m r_{0}^{2} / \epsilon_{0}\right)^{1 / 2}$ and length in units of $r_{0}$. In these units, Eq. (A2) takes the form

$$
\begin{aligned}
\ddot{\mathbf{r}}_{\mathbf{n}}= & -\bar{\eta}\left(\dot{\mathbf{r}}_{\mathbf{n}}-\dot{\mathbf{X}}\right)+\bar{K}_{x} \cdot\left(\mathbf{r}_{\mathbf{n}+\hat{x}}+\mathbf{r}_{\mathbf{n}-\hat{x}}-2 \mathbf{r}_{\mathbf{n}}\right) \\
& +\bar{K}_{y} \cdot\left(\mathbf{r}_{\mathbf{n}+\hat{y}}+\mathbf{r}_{\mathbf{n}-\hat{y}}-2 \mathbf{r}_{\mathbf{n}}\right) \\
& +\bar{K}_{1} \cdot\left[\mathbf{X}+\mathbf{n} a+h_{\mathbf{n}} \hat{z}-\mathbf{r}_{\mathbf{n}}-W \hat{z}\right]+\overline{\mathbf{F}}_{\mathbf{n}}+\overline{\mathbf{f}}_{\mathbf{n}},
\end{aligned}
$$

where $\bar{\eta}=\eta \tau, \bar{K}_{x}=K_{x} r_{0}^{2} / \epsilon_{0}$ (and similar for $\bar{K}_{y}$ and $\bar{K}_{1}$ ), $\overline{\mathbf{F}}=\mathbf{F} r_{0} / \epsilon_{0}$ and $\overline{\mathbf{f}}=\mathbf{f} r_{0} / \epsilon_{0}$. Note also that Eq. (A7) takes the form

$$
\left\langle\bar{f}_{\mathbf{n}}^{i}(t) \bar{f}_{\mathbf{n}^{\prime}}^{j}\left(t^{\prime}\right)\right\rangle=2 \bar{\eta} \bar{T} \delta\left(t-t^{\prime}\right) \delta_{i j} \delta_{\mathbf{n n}^{\prime}},
$$

where $\bar{T}=k_{B} T / \epsilon_{0}$ and where time $t$ is measured in units of $\tau$.

\section{Derivation of $\boldsymbol{\eta}$}

We have shown elsewhere that when an adsorbed atom vibrates, it will experience a damping due to emission of lattice waves. ${ }^{12}$ We can apply the same theory to the present case, where the adatoms are replaced by an atom in the bottom layer of the block (or top layer of the substrate). If we treat the atom as an Einstein oscillator with the characteristic resonance frequency $\omega_{0}$, then

$$
\eta \approx \frac{m_{0} \omega_{0}^{4}}{8 \rho c_{T}^{3}}
$$

where $\rho=m_{0} / a^{3}$ is the mass density and $\omega_{0}^{2} \approx 2 k_{0} / m_{0}$. Using $c_{T}^{2} \approx k_{0} a^{2} / m_{0}$ gives $\eta \approx c_{T} / 2 a$. More generally one may write $\eta=A c_{T} / a$ where $A$ is a constant of order unity. Note that since $\omega_{0} \approx c_{T} / a$ the oscillator is overdamped.

\section{Calculation of the effective corrugation}

If we write

$$
\mathbf{r}_{\mathbf{n}}=\mathbf{n} a+\mathbf{u}(\mathbf{n}),
$$

then, if $\mathbf{u}(\mathbf{n})$ varies slowly with $\mathbf{n}$, we can treat $\mathbf{n}$ as a continuous variable and expand

$$
\mathbf{u}(\mathbf{n}+\hat{x})=\mathbf{u}(\mathbf{n})+\frac{\partial \mathbf{u}}{\partial n_{x}}+\frac{1}{2} \frac{\partial^{2} \mathbf{u}}{\partial n_{x}^{2}}+\ldots,
$$

where the derivatives are calculated in the point $\mathbf{n}$. If we expand $\mathbf{u}(\mathbf{n}+\hat{y})$ in the same way and substitute the results in Eq. (A2) we get for a stationary case,

$$
\begin{aligned}
& K_{x} \cdot \frac{\partial^{2} \mathbf{u}}{\partial n_{x}^{2}}+K_{y} \cdot \frac{\partial^{2} \mathbf{u}}{\partial n_{y}^{2}}+K_{1} \cdot\left(\left[z_{0}-h_{0} \cos (q x)\right] \hat{z}-\mathbf{u}-W \hat{z}\right) \\
& \quad+\mathbf{F}_{\mathbf{n}}=0 .
\end{aligned}
$$

Since $x=n_{x} a$, the $z$-component of this equation takes the form

$$
a^{2} k_{0 B} \frac{\partial^{2} u_{z}}{\partial x^{2}}+k_{1}\left[z_{0}-h_{0} \cos (q x)-u_{z}-W\right]=-F_{z}(x) .
$$

In the present case $F_{z}=0$ so that the solution to this equation is

$$
u_{z}=u_{1}+u_{2} \cos (q x),
$$

where $u_{1}$ and $u_{2}$ are constants given by

$$
\begin{aligned}
& u_{1}=z_{0}-W, \\
& u_{2}=-\frac{h_{0}}{1+\left(k_{0 B} / k_{1}\right)(q a)^{2}} .
\end{aligned}
$$

In this article $q=2 \pi / L_{x}$ and with $k_{0 B}=G a$ and $k_{1}$ $=E a^{2} / W_{1} \quad$ we get $\quad\left(k_{0 B} / k_{1}\right)(q a)^{2}=\left[2 \pi^{2} /(1+\nu)\right]$ $\times\left(a W_{1} / L_{x}^{2}\right)$. In the simulations in this article $L_{x} / a=150$ or 200, $W_{1}=100 \AA$, and $2.1 \AA<a<4.4 \AA$, so that $\left(k_{0 B} / k_{1}\right)$ $\times(q a)^{2} \approx 0.01-0.02$, i.e., to within one or two percent, the lower free surface of the elastic slab will follow the rigid profile to which the upper surface has been "glued."

\section{Pressure distribution}

Let us assume that the cylindrical asperity described by Eq. (A13) is squeezed against a flat rigid substrate. This will give rise to contact for $L_{x} / 2-l<x<L_{x} / 2+l$, where $2 l$ denote the width (in the $x$-direction) of the contact area. It is convenient to introduce $\bar{x}=x-L_{x} / 2$. In this section we calculate the pressure distribution at the interface under the assumption that $q l \ll 1$. In this case we can expand $\cos (q x)$ $=-\cos (q \bar{x}) \approx-\left[1-(q \bar{x})^{2} / 2\right]$ so that Eq. (A13) takes the form 


$$
a^{2} k_{0 B} \frac{\partial^{2} u_{z}}{\partial \bar{x}^{2}}+k_{1}\left(z_{0}+h_{0}\left[1-(q \bar{x})^{2} / 2\right]-u_{z}-W\right)=-F_{z} .
$$

In the contact region $-l<\bar{x}<l$ we have $u_{z}=$ const $=u_{0}$ so that Eq. (A14) takes the form

$$
k_{1}\left(z_{0}+h_{0}\left[1-(q \bar{x})^{2} / 2\right]-u_{0}-W\right)=-F_{z} .
$$

Thus it follows that

$$
F_{z}=a^{2} \sigma(\bar{x})
$$

where the perpendicular stress or pressure

$$
\sigma(\bar{x})=\sigma_{0}\left(1-\bar{x}^{2} / l^{2}\right),
$$

with

$$
\sigma_{0}=\left(k_{1} l^{2} / 2 a^{2}\right) h_{0} q^{2},
$$

and

$$
h_{0}(q l)^{2}=2\left(h_{0}+z_{0}-u_{0}-W\right) .
$$

Note that the average pressure over the contact area is $2 / 3$ of the maximum $\left(\sigma_{0}\right)$ pressure. This stress averaged over the whole region $0<x<L_{x}$ equals

$$
P=\frac{1}{L_{x}} \int_{l}^{l} d \bar{x} \sigma(\bar{x})=\frac{4 \sigma_{0} l}{3 L_{x}} .
$$

Combining Eqs. (A16) and (A17) and using $k_{1}=E a^{2} / W$ gives

$$
\sigma_{0}=\left(\frac{9 h_{0}}{8 W} \pi^{2} P^{2} E\right)^{1 / 3} .
$$

\section{Numerical implementation}

The equations of motion are integrated by the velocity version of the Verlet algorithm. ${ }^{13}$ The time step is set to $0.001 \tau$ where $\tau=\left(m r_{0}^{2} / \epsilon_{0}\right)^{1 / 2}$, giving a very good conserva- tion of the total energy when the fluctuating external force is set to zero. To enhance the efficiency of the implementation, and to improve its long time stability, the Lennard-Jones potentials used in the simulations are truncated at $r=3.5 r_{0}$, and replaced by a cubic polynomial for $3.5<r / r_{0}<4$. The potentials are assumed to be identically zero for $r>4 r_{0}$. The coefficients of the polynomial are selected in such a way that each resulting potential is everywhere continuous with its first derivative. The Cartesian components of the stochastic force with Gaussian distribution, entering Eq. (A2), is generated by the algorithm described in Ref. 14 .

${ }^{1}$ B. N. J. Persson, Sliding Friction: Physical Principles and Applications (Springer, Heidelberg, 1998); Surf. Sci. Rep. 33, 83 (1999); J. Krim, Sci. Am. 275, 74 (1996).

${ }^{2}$ J. Gao, W. D. Luedtke, and U. Landman, Phys. Rev. Lett. 79, 705 (1997); J. Phys. Chem. B 101, 4013 (1997).

${ }^{3}$ J. N. Israelachvili, Intermolecular and Surface Forces (Academic, London, 1995); M. L. Gee, P. M. McGuiggan, and J. N. Israelachvili, J. Chem. Phys. 93, 1895 (1990).

${ }^{4}$ B. N. J. Persson (unpublished).

${ }^{5}$ L. Demirel and S. Granick, Phys. Rev. Lett. 77, 2261 (1996); J. Chem. Phys. 106, 6889 (1998).

${ }^{6}$ J. Klein and E. Kumacheva, Science 269, 816 (1995); J. Chem. Phys. 108, 6996 (1998); 108, 7010 (1998).

${ }^{7}$ B. N. J. Persson and E. Tosatti, Phys. Rev. B 50, 5590 (1994).

${ }^{8} \mathrm{~J}$. N. Israelachvili (private communication).

${ }^{9}$ F. Mugele and M. Salmeron, Phys. Rev. Lett. (submitted).

${ }^{10}$ Figure 14 gives the average pressure, i.e., the load divided by the area $L_{x} L_{y}$, and this average pressure is much smaller than the maximum pressure in the contact area.

${ }^{11} \mathrm{~S}$. Aubry, in Solitons in Condensed Matter, edited by A. R. Bishop and T. Schneider (Springer, Berlin, 1978), p. 264; W. Selke, in Phase Transitions and Critical Phenomena, edited by C. Domb and J. L. Lebowitz (Academic, London, 1992), Vol. 15, p. 1; B. N. J. Persson, Surf. Sci. Rep. 15, 1 (1992).

${ }^{12}$ B. N. J. Persson and R. Ryberg, Phys. Rev. B 32, 3586 (1985).

${ }^{13}$ M. P. Allen and D. J. Tildesly, Computer Simulation of Liquids (Clarendon, Oxford, 1989).

${ }^{14}$ W. H. Press, B. P. Flannery, S. A. Teukolsky, and W. T. Vetterling, Numerical Recipes (Cambridge University Press, Cambridge, 1986), Sec. 7.2. 